\title{
Linear Rayleigh and Raman scattering to the second order: Analytical results for light scattering by any scatterer of size $k_{0} d \lesssim 1 / 10$
}

\author{
Robert P. Cameron* \\ Department of Physics, University of Strathclyde, Glasgow G4 ONG, United Kingdom and \\ School of Physics and Astronomy, University of Glasgow, Glasgow G12 8QQ, United Kingdom \\ Neel Mackinnon \\ School of Physics and Astronomy, University of Glasgow, Glasgow G12 8QQ, United Kingdom
}

(Dated: July 2, 2018)

\begin{abstract}
We extend the usual multipolar theory of linear Rayleigh and Raman scattering to include the second-order correction. The new terms promise a wealth of information about the shape of a scatterer and yet are insensitive to the scatterer's chirality. Our extended theory might prove especially useful for analysing samples in which the scatterers have non-trivial shapes but no chiral preference overall, as the zeroth-order theory offers little information about shape and the first-order correction is often quenched for such samples. A basic estimate suggests that our extended theory can be applied to a scatterer as large as $k_{0} d \sim 1 / 10$ with less than $\sim 0.1 \%$ error resulting from the neglect of the third- and higher-order corrections. Our results are entirely analytical.
\end{abstract}

\section{INTRODUCTION}

Light scattering is an all-pervasive phenomenon. Together with light absorption, it is largely responsible for the appearance of the material world $[1,2]$. Theoretical understanding of light scattering is sufficiently advanced to enable determinations of the nature of interstellar dust [3], radar [4], studies of the structures of viruses [5] and the measurement of the salinity of seawater [6], to name but a few applications. There is much still to be explored, however, and the study of light scattering remains at the cutting edge of research [7-15].

One can distinguish between different types of light scattering $[1,16-21]$. This paper is concerned with one of the simplest and most common of these: a two-photon process in which a quantum of light collides with an electrically neutral scatterer [22]. Nonlinear light scattering processes, involving three or more photons, are also possible and are proving increasingly useful $[8,9,22]$. We do not consider these here, however: our interest is emphatically in linear light scattering.

If the aforementioned collision is elastic, it is usually referred to as Rayleigh scattering for $k_{0} d \ll 1[16,23,24]$ or Willis-Tyndall scattering for $1 / 10<k_{0} d<10$ [25], with $d$ a characteristic length of the scatterer and $k_{0}$ the wavenumber of the incident photon. If the collision is inelastic [19], it is referred to as combination [20] or Raman scattering [21]. For $k_{0} d \ll 1$, light scattering is often treated using the zeroth-order Rayleigh theory (or its extension to Raman scattering), in which multipolar expansions for the scatterer are truncated at electric-dipole order. The first-order correction to this theory was introduced relatively recently $[26,27]$ and has proved extremely useful in the laboratory for the analysis of chiral molecules $[2,5,28-30]$, as it discriminates between left

\footnotetext{
* robert.p.cameron@strath.ac.uk
}

and right. For $1 / 10<k_{0} d<10$, elastic light scattering by scatterers with sufficiently simple shapes can be treated using the mathematical machinery of (analytical) Mie theory [1, 25]. Numerical approaches are usually used instead for scatterers with more complicated shapes [3], although a semi-analytical extension of Mie theory has recently been put forward $[7,13]$.

In this paper we introduce the second-order correction to the zeroth-order Rayleigh / Raman theory. The new terms promise a wealth of information about the shape of a scatterer and yet are insensitive to the scatterer's chirality (left versus right) if the scatterer happens to be chiral. Our extended theory might prove especially useful for analysing samples in which the scatterers have nontrivial shapes but no chiral preference overall. Consider, for example, a racemic sample of chiral molecules, as might be produced in a symmetric chemical reaction using achiral precursors [31]. The zeroth-order theory offers little information about the shapes of the molecules and the first-order correction is quenched by virtue of there being equal numbers of left- and right-handed molecules. The second-order correction, however, can still be exploited as an incisive probe of the shapes of the molecules. Our extended theory might also help bridge the perceived divide between small scatterers $\left(k_{0} d \ll 1\right)$ and mediumsized scatterers $\left(1 / 10<k_{0} d<10\right)$ : a basic estimate suggests that the zeroth-order theory together with its firstand second-order corrections can be applied to a scatterer as large as $k_{0} d \sim 1 / 10$, with less than $\sim 0.1 \%$ error resulting from the neglect of the third- and higher-order corrections. Let us emphasise here that our results are entirely analytical.

In what follows we imagine ourselves to be in an inertial frame of reference with time $t$; right-handed Cartesian coordinates $x, y$ and $z$ with associated unit vectors $\hat{\mathbf{x}}, \hat{\mathbf{y}}$ and $\hat{\mathbf{z}}$ and spherical coordinates $r, \theta$ and $\phi$ with associated unit vectors $\hat{\mathbf{r}}, \hat{\boldsymbol{\theta}}$ and $\hat{\boldsymbol{\phi}}$. The SI system of units is adopted and the Einstein summation convention [32] 
is to be understood, with subscripts $a, b, c, \ldots$ running over $x, y$ and $z$. Complex quantities are indicated using tildes, except where otherwise stated.

\section{GENERAL CALCULATION}

Our aim in this paper is to introduce the secondorder correction to the zeroth-order Rayleigh / Raman theory as simply as possible. We focus our attention, therefore, upon a semiclassical model in which a single scatterer is illuminated by weak, quasi-monochromatic light that has been 'switched on' slowly at some distant time in the past. The scatterer could represent a small molecule in vacuum [2], for example. We make no specific assumptions about the scatterer, except that it is smaller than around one-tenth of the wavelength of the illuminating light and is localised near the spatial origin $(x=y=z=0)$, so that we can perform converging multipolar expansions about the spatial origin. The relationship of the 'local multipole approach' [26, 27] used in this paper to the 'distributed dipole approach' [3] is examined in [33].

The electric and magnetic fields of the illuminating light at the spatial origin have the following forms:

$$
\begin{aligned}
& \mathbf{E} \approx \mathfrak{R}\left(\tilde{\mathbf{E}} \mathrm{e}^{-\mathrm{i} \omega_{0} t}\right) \\
& \mathbf{B} \approx \mathfrak{R}\left(\tilde{\mathbf{B}} \mathrm{e}^{-\mathrm{i} \omega_{0} t}\right),
\end{aligned}
$$

with $\omega_{0}=c k_{0}$ the angular frequency of the illuminating light. The illuminating light induces oscillations in the charge and current distributions of the scatterer and these oscillations are themselves the source of electromagnetic radiation: scattered light. The electric and magnetic fields of the scattered light have the following forms:

$$
\begin{aligned}
& \mathbf{e} \approx \mathfrak{R}\left(\tilde{\mathbf{e}} \mathrm{e}^{-\mathrm{i} \omega t}\right) \\
& \mathbf{b} \approx \mathfrak{R}\left(\tilde{\mathbf{b}} \mathrm{e}^{-\mathrm{i} \omega t}\right),
\end{aligned}
$$

with $\omega=c k$ the angular frequency of the scattered light. This is equal to $\omega_{0}$ for Rayleigh scattering, or $\omega_{0}-\omega_{f i}$ for a Raman-scattering transition $f \leftarrow i$, where $\omega_{f i}$ is the angular frequency of the transition. In this paper we use a parameter $\lambda=1$ to help us keep track of order in our multipolar expansions. The powers of $\lambda$ quoted by us have their origins in Taylor expansions. Each term in one of these expansions has an additional spatial derivative (of the illuminating light or scattered light) and length scale (the position of some constituent of the scatterer relative to the chosen origin of multipolar expansion) relative to the term before it. We thus associate each power of $\lambda$ with a factor of $\sim\left(k_{0} d\right) \sim(k d) \lesssim(1 / 10)$, where $d$ is a characteristic length of the scatterer, as above. Far from the scatterer $(k r \gg 1)$,

$$
\begin{aligned}
\tilde{e}_{a} & \approx \frac{\mu_{0} \omega^{2} \mathrm{e}^{\mathrm{i} k r}}{4 \pi r}\left(\delta_{a b}-\hat{r}_{a} \hat{r}_{b}\right)\left(\lambda^{0} \tilde{\mu}_{b}\right. \\
& +\lambda^{1} \frac{1}{c} \epsilon_{b c d} \tilde{M}_{c} \hat{r}_{d}-\lambda^{1} \frac{\mathrm{i} k}{3} \tilde{\Theta}_{b c} \hat{r}_{c} \\
& \left.-\lambda^{2} \frac{\mathrm{i} k}{2 c} \epsilon_{b c d} \tilde{M}_{c e} \hat{r}_{e} \hat{r}_{d}-\lambda^{2} \frac{k^{2}}{6} \tilde{Q}_{b c d} \hat{r}_{c} \hat{r}_{d}\right) \\
\tilde{b}_{a} & \approx \frac{1}{c} \epsilon_{a b c} \hat{r}_{b} \tilde{e}_{c},
\end{aligned}
$$

where $\tilde{\mu}_{a}, \tilde{\Theta}_{a b}$ and $\tilde{Q}_{a b c}$ are the induced electric-dipole, electric-quadrupole and electric-octupole moments of the scatterer, and $\tilde{M}_{a}$ and $\tilde{M}_{a b}$ are the induced magneticdipole and magnetic-quadrupole moments. These results, (5) and (6), constitute an extension of those given in [2] to include terms of order $\lambda^{2}$ and can be regarded as a special case of the results given in [34], particular to harmonic oscillations. The induced multipole moments of the scatterer are related to the illuminating light by the scatterer's property tensors:

$$
\begin{aligned}
\lambda^{0} \tilde{\mu}_{a} & \approx \lambda^{0} \tilde{\alpha}_{a b} \tilde{E}_{b}+\lambda^{1} \frac{1}{3} \tilde{A}_{a, b c} \partial_{c} \tilde{E}_{b}+\lambda^{1} \tilde{G}_{a b} \tilde{B}_{b} \\
& +\lambda^{2} \frac{1}{6} \tilde{B}_{a, b c d} \partial_{d} \partial_{c} \tilde{E}_{b}+\lambda^{2} \frac{1}{2} \tilde{D}_{a, b c}^{(m)} \partial_{c} \tilde{B}_{b} \\
\lambda^{1} \tilde{M}_{a} & \approx \lambda^{1} \tilde{\mathscr{G}}_{a b} \tilde{E}_{b}+\lambda^{2} \frac{1}{3} \tilde{D}_{a, b c} \partial_{c} \tilde{E}_{b}+\lambda^{2} \tilde{\chi}_{a b} \tilde{B}_{b} \\
\lambda^{1} \tilde{\Theta}_{a b} & \approx \lambda^{1} \tilde{\mathscr{A}}_{c, a b} \tilde{E}_{c}+\lambda^{2} \tilde{C}_{a b, c d} \partial_{d} \tilde{E}_{c}+\lambda^{2} \tilde{\mathscr{D}}_{c, a b} \tilde{B}_{c} \\
\lambda^{2} \tilde{M}_{a b} & \approx \lambda^{2} \tilde{\mathscr{D}}_{c, a b}^{(m)} \tilde{E}_{c} \\
\lambda^{2} \tilde{Q}_{a b c} & \approx \lambda^{2} \tilde{\mathscr{B}}_{d, a b c} \tilde{E}_{d} .
\end{aligned}
$$

These results, (7)-(11), also constitute an extension of those given in [2] to include terms of order $\lambda^{2}$. Explicit quantum-mechanical expressions for multipole moments and property tensors are given in appendix A, where we also show how the property tensors reduce under certain special circumstances.

We consider the Stokes parameters $s_{\xi}(\xi \in\{0,1,2,3\})$ of the scattered light, which can be written succinctly as follows:

$$
s_{\xi}=\tilde{f}_{\xi a b} \tilde{e}_{a} \tilde{e}_{b}^{*}
$$

with

$$
\begin{aligned}
\tilde{f}_{0 a b} & =\hat{\theta}_{a} \hat{\theta}_{b}+\hat{\phi}_{a} \hat{\phi}_{b}, \\
\tilde{f}_{1 a b} & =\hat{\theta}_{a} \hat{\theta}_{b}-\hat{\phi}_{a} \hat{\phi}_{b}, \\
\tilde{f}_{2 a b} & =-\hat{\theta}_{a} \hat{\phi}_{b}-\hat{\phi}_{a} \hat{\theta}_{b} \\
\tilde{f}_{3 a b} & =-\mathrm{i} \hat{\theta}_{a} \hat{\phi}_{b}+\mathrm{i} \hat{\phi}_{a} \hat{\theta}_{b} .
\end{aligned}
$$

Note that $\tilde{f}_{\xi a b}=\tilde{f}_{\xi b a}^{\star}$, which ensures that the $s_{\xi}$ are real. Furthermore $\tilde{f}_{0 a b}, \tilde{f}_{1 a b}$, and $\tilde{f}_{2 a b}$ are symmetric in $a$ and $b$ and purely real whereas $\tilde{f}_{3 a b}$ is antisymmetric in $a$ and $b$ and purely imaginary. 
Working to order $\lambda^{2}$, we substitute (5) with (7)-(11) into (12) and find that the Stokes parameters of the scattered light take the following form:

$$
s_{\xi} \approx \lambda^{0} s_{\xi}^{(0)}+\lambda^{1} s_{\xi}^{(1)}+\lambda^{2} s_{\xi}^{(2)}
$$

with the $s_{\xi}^{(0)}$, the $s_{\xi}^{(1)}$ and the $s_{\xi}^{(2)}$ as defined below.

The zeroth-order theory is embodied by the following:

$$
s_{\xi}^{(0)}=s_{\xi}^{\alpha-\alpha},
$$

with

$$
s_{\xi}^{\alpha-\alpha}=K \Re\left(\frac{1}{2} \tilde{\alpha}_{a b} \tilde{\alpha}_{c d}^{*} \tilde{f}_{\xi a c} \tilde{E}_{b} \tilde{E}_{d}^{*}\right)
$$

and

$$
K=\frac{\mu_{0}^{2} \omega^{4}}{8 \pi^{2} r^{2}},
$$

as is well known $[2,22]$. The zeroth-order theory is already sufficient to account in a basic way for the polarisation, depolarisation and colour of the light from the sky [16, 23, 24], for example.

The first-order correction is due to interference between light waves scattered via the familiar property tensor ' $\alpha$ ' and light waves scattered via the optical activity property tensors ' $A$ ' and ' $G$ ', as embodied by the following:

$$
s_{\xi}^{(1)}=s_{\xi}^{\alpha-A}+s_{\xi}^{\alpha-G}
$$

with

$$
\begin{aligned}
s_{\xi}^{\alpha-A} & =K \Re\left(\frac{1}{3} \tilde{\alpha}_{a b} \tilde{A}_{c, d e}^{*} \tilde{f}_{\xi a c} \tilde{E}_{b} \partial_{d} \tilde{E}_{e}^{*}\right. \\
& \left.+\frac{\mathrm{i} k}{3} \tilde{\alpha}_{a b} \tilde{\mathscr{A}}_{c, d e}^{*} \tilde{f}_{\xi a d} \tilde{E}_{b} \tilde{E}_{c}^{*} \hat{r}_{e}\right) \\
s_{\xi}^{\alpha-G} & =K \Re\left(\tilde{\alpha}_{a b} \tilde{G}_{c d}^{*} \tilde{f}_{\xi a c} \tilde{E}_{b} \tilde{B}_{d}^{*}\right. \\
& \left.+\frac{1}{c} \tilde{\alpha}_{a b} \tilde{\mathscr{G}}_{c d}^{*} \epsilon_{e c f} \tilde{f}_{\xi a e} \tilde{E}_{b} \tilde{E}_{d}^{*} \hat{r}_{f}\right),
\end{aligned}
$$

as is also well known [2, 22]. The first-order correction accounts for the leading-order contributions to optical activity in an isotropic sample of chiral molecules [26, 27]. Natural Raman optical activity has been developed into an incisive spectroscopic tool for chiral scatterers both large and small [2, 5, 28-30]. In contrast, natural Rayleigh optical activity has been reported for a handful of large chiral biological structures, including octopus sperm [35], but has thus far proved elusive for small chiral molecules [2], in spite of potential applications such as the robust assignment of absolute configuration [36]. The difficulties here might be partially overcome using structured light [37, 38]. Interestingly, orientated achiral molecules can also exhibit natural optical activity via the first-order correction, embodied by the $s_{\xi}^{(1)}[2]$, and partially orientated chiral molecules can exhibit natural optical activity via the zeroth-order theory, embodied by the $s_{\xi}^{(0)}[39]$.

We find that the second-order correction is due to mutual interference between light waves scattered via the optical activity property tensors ' $A$ ' and ' $G$ ', together with equally important contributions due to interference between light waves scattered via the familiar property tensor ' $\alpha$ ' and light waves scattered via the more exotic property tensors ' $B$ ', ' $C$ ', ' $D$ ', ' $D^{m}$ ' and ' $\chi$ ', as embodied by the following:

$$
\begin{aligned}
s_{\xi}^{(2)} & =s_{\xi}^{A-A}+s_{\xi}^{G-A}+s_{\xi}^{G-G} \\
& +s_{\xi}^{\alpha-B}+s_{\xi}^{\alpha-C}+s_{\xi}^{\alpha-D}+s_{\xi}^{\alpha-D^{m}}+s_{\xi}^{\alpha-\chi},
\end{aligned}
$$

with

$$
\begin{aligned}
s_{\xi}^{A-A} & =K \Re\left(\frac{1}{18} \tilde{A}_{a, b c} \tilde{A}_{d, e f}^{*} \tilde{f}_{a d} \partial_{b} \tilde{E}_{c} \partial_{e} \tilde{E}_{f}^{*}\right. \\
& +\frac{\mathrm{i} k}{9} \tilde{A}_{a, b c} \tilde{\mathscr{A}}_{d, e f}^{*} \tilde{f}_{a e} \partial_{b} \tilde{E}_{c} \tilde{E}_{d}^{*} \hat{r}_{f} \\
& \left.+\frac{k^{2}}{18} \tilde{\mathscr{A}}_{a, b c} \tilde{\mathscr{A}}_{d, e f}^{*} \tilde{E}_{a} \tilde{f}_{\xi b e} \hat{r}_{c} \tilde{E}_{d}^{*} \hat{r}_{f}\right), \\
s_{\xi}^{G-A} & =K \Re\left(\frac{1}{3} \tilde{G}_{a b} \tilde{A}_{c, d e}^{*} \tilde{f}_{\xi a c} \tilde{B}_{b} \partial_{d} \tilde{E}_{e}^{*}\right. \\
& +\frac{1}{3 c} \tilde{\mathscr{G}}_{a b} \tilde{A}_{c, d e}^{*} \epsilon_{f a g} \hat{r}_{g} \tilde{E}_{b} \partial_{d} \tilde{E}_{e}^{*} \tilde{f}_{\xi f c} \\
& +\frac{\mathrm{i} k}{3} \tilde{G}_{a b} \tilde{\mathscr{A}}_{c, d e}^{*} \tilde{f}_{\xi a d} \tilde{B}_{b} \tilde{E}_{c}^{*} \hat{r}_{e} \\
& \left.+\frac{\mathrm{i} k}{3 c} \tilde{\mathscr{G}}_{a b} \tilde{\mathscr{A}}_{c, d e}^{*} \epsilon_{f a g} \tilde{f}_{\xi f d} \hat{r}_{g} \tilde{E}_{b} \tilde{E}_{c}^{*} \hat{r}_{e}\right) \\
s_{\xi}^{G-G} & =K \Re\left(\frac{1}{2} \tilde{G}_{a b} \tilde{G}_{c d}^{*} \tilde{f}_{\xi a c} \tilde{B}_{b} \tilde{B}_{d}^{*}\right. \\
& +\frac{1}{c} \tilde{G}_{a b} \tilde{\mathscr{G}}_{c d}^{*} \tilde{f}_{\xi a e} \epsilon_{e c f} \tilde{B}_{b} \tilde{E}_{d}^{*} \hat{r}_{f} \\
& \left.\tilde{\mathscr{G}}_{a b} \tilde{\mathscr{G}}_{c d}^{*} \epsilon_{a e f} \epsilon_{c g h} \tilde{f}_{\xi e g} \tilde{E}_{b} \tilde{E}_{d}^{*} \hat{r}_{f} \hat{r}_{h}\right)
\end{aligned}
$$


the optical activity cross terms and

$$
\begin{aligned}
s_{\xi}^{\alpha-B} & =K \Re\left(\frac{1}{6} \tilde{\alpha}_{a b} \tilde{B}_{c, d e f}^{*} \tilde{f}_{\xi a c} \tilde{E}_{b} \partial_{d} \partial_{e} \tilde{E}_{f}^{*}\right. \\
& \left.-\frac{k^{2}}{6} \tilde{\alpha}_{a b} \tilde{\mathscr{B}}_{c, d e f}^{*} \tilde{f}_{\xi a d} \tilde{E}_{b} \tilde{E}_{c}^{*} \hat{r}_{e} \hat{r}_{f}\right), \\
s_{\xi}^{\alpha-C} & =K \Re\left(\frac{\mathrm{i} k}{3} \tilde{\alpha}_{a b} \tilde{C}_{c d, e f}^{*} \tilde{f}_{\xi a c} \tilde{E}_{b} \hat{r}_{d} \partial_{e} \tilde{E}_{f}^{*}\right), \\
s_{\xi}^{\alpha-D} & =K \Re\left(\frac{\mathrm{i} k}{3} \tilde{\alpha}_{a b} \tilde{\mathscr{D}}_{c, d e}^{*} \tilde{f}_{\xi a d} \tilde{E}_{b} \tilde{B}_{c}^{*} \hat{r}_{e}\right. \\
& \left.+\frac{1}{3 c} \tilde{\alpha}_{a b} \tilde{D}_{c, d e}^{*} \tilde{f}_{\xi a f} \tilde{E}_{b} \partial_{d} \tilde{E}_{e}^{*} \epsilon_{f c g} \hat{r}_{g}\right) \\
s_{\xi}^{\alpha-D^{m}} & =K \Re\left(\frac{1}{2} \tilde{\alpha}_{a b} \tilde{D}_{c, d e}^{(m) *} \tilde{f}_{\xi a c} \tilde{E}_{b} \partial_{e} \tilde{B}_{d}^{*}\right. \\
& \left.+\frac{\mathrm{i} k}{2 c} \tilde{\alpha}_{a b} \tilde{\mathscr{D}}_{c, d e}^{(m) *} \tilde{f}_{\xi a f} \tilde{E}_{b} \tilde{E}_{c}^{*} \epsilon_{f d g} \hat{r}_{e} \hat{r}_{g}\right) \\
s_{\xi}^{\alpha-\chi} & =K \Re\left(\frac{1}{c} \tilde{\alpha}_{a b} \tilde{\chi}_{c d}^{*} \tilde{f}_{\xi a e} \tilde{E}_{b} \tilde{B}_{d}^{*} \epsilon_{e c f} \hat{r}_{f}\right)
\end{aligned}
$$

the exotic interference terms. The second-order correction does not appear to have been described explicitly before and is the central result of this paper. Accounted for by the $s_{\xi}^{(2)}$ are the "terms in $G^{2}$ and $A^{2}$ " alluded to in [2]. Light scattering to second order has also been touched upon in [33], where the possibility of new rotational Raman lines with zero background is highlighted.

It is important to note that each of the optical activity property tensors ' $A$ ' and ' $G$ ' and each of the exotic property tensors ' $B$ ', ' $C$ ', ' $D$ ', ' $D^{m}$ ' and ' $\chi$ ' is implicitly dependent upon our choice of origin for multipolar expansions: they differ when calculated about different origins. In appendix B we show that our physical predictions (based upon the complete Stokes parameters $s_{\xi}$ of the scattered light, with all terms of order $\lambda^{0}, \lambda^{1}$ and $\lambda^{2}$ considered simultaneously) are nevertheless independent of our choice of origin for multipolar expansions, as they should be.

As mentioned earlier, a basic estimate based on the orders of the Taylor expansions reveals that $s_{\xi}^{(0)} \sim$ $\left(k_{0} d\right)^{-1} s_{\xi}^{(1)} \sim\left(k_{0} d\right)^{-2} s_{\xi}^{(2)} \ldots$ This suggests that even for a scatterer with $k_{0} d \sim 1 / 10$, the second-order correction will yield a modification of only 1\% to the zeroth-order theory. That is to say, exploitation of the second-order correction in the laboratory will demand precision measurements. This estimate also implies that neglect of the third- and higher-order corrections gives rise to $\lesssim 0.1 \%$ error for $k_{0} d \lesssim 1 / 10$, which suggests that the zeroth-order theory together with its first- and second-order corrections might serve as a precise alternative to numerical approaches for $k_{0} d \lesssim 1 / 10$.

To better appreciate the validity of such estimates, note first the well-established fact $[2,5,28-30,36]$ that the first-order correction, which has contributions of the form ' $\alpha \times A$ ' and ' $\alpha \times G$ ', is typically smaller than the zeroth-order theory, which has contributions of the form ' $\alpha \times \alpha$ ', by the predicted factor of $\sim\left(k_{0} d\right)$. It follows immediately (from ' $A \sim\left(k_{0} d\right) \alpha$ ' and ' $G \sim\left(k_{0} d\right) \alpha$ ') that the optical activity cross terms, which have contributions of the form ' $A \times A$ ', ' $A \times G$ ' and ' $G \times G$ ', will typically be smaller than the first-order correction by the same factor of $\sim\left(k_{0} d\right)$. One expects the order of magnitude of the exotic interference terms to be similar because a change in the choice of origin for mutlipolar expansion intermixes these with the optical activity cross terms as shown in appendix B. Preliminary calculations performed by us using a dynamic coupling model [40] support these claims.

The results we have given thus far are rather general. They might be applied to Rayleigh or Raman scattering, on or off resonance, for any scatterer in any orientation. Moreover, they can be extended to account for the presence of static fields, by considering distortions of the property tensors $[2,22]$. Let us also emphasise that our results can be applied for different forms of (quasimonochromatic) illuminating light: plane-wave illumination, considered below, is but one possibility. Illumination by more exotic forms of light could open the door to new possibilities, one example of which is highlighted in section IV. Evanescent fields play important roles in scattering-type near-field optical microscopy techniques [10] and it has recently been shown that illumination by standing waves yields new possibilities for optical activity $[37,38]$, to give two more examples of non-plane-wave illumination in light scattering.

\section{PLANE-WAVE ILLUMINATION AND ROTATIONAL AVERAGING}

A scattering experiment often involves a Gaussian beam of light illuminating a fluid sample. The Stokes parameters $s_{\xi}$ of the scattered light are measured as a function of the Stokes parameters $S_{\xi}(\xi \in\{0,1,2,3\})$ of the illuminating light and the scattering angle $\theta$. With such a setup in mind, we now consider the following specific example.

With regards to the illuminating light, we consider a plane wave propagating in the $+z$ direction:

$$
\begin{aligned}
\tilde{\mathbf{E}} & =\left(\tilde{E}_{0 x} \hat{\mathbf{x}}+\tilde{E}_{0 y} \hat{\mathbf{y}}\right) \\
\tilde{\mathbf{B}} & =\frac{1}{c}\left(-\tilde{E}_{0 y} \hat{\mathbf{x}}+\tilde{E}_{0 x} \hat{\mathbf{y}}\right) .
\end{aligned}
$$

We define the Stokes parameters $S_{\xi}$ of the illuminating light as follows:

$$
\begin{aligned}
& S_{0}=\tilde{E}_{0 x} \tilde{E}_{0 x}^{*}+\tilde{E}_{0 y} \tilde{E}_{0 y}^{*}, \\
& S_{1}=\tilde{E}_{0 x} \tilde{E}_{0 x}^{*}-\tilde{E}_{0 y} \tilde{E}_{0 y}^{*}, \\
& S_{2}=-\left(\tilde{E}_{0 x} \tilde{E}_{0 y}^{*}+\tilde{E}_{0 y} \tilde{E}_{0 x}^{*}\right) \\
& S_{3}=-\mathrm{i}\left(\tilde{E}_{0 x} \tilde{E}_{0 y}^{*}-\tilde{E}_{0 y} \tilde{E}_{0 x}^{*}\right) .
\end{aligned}
$$


We also average over all possible orientations of the scatterer, with this average denoted using angular brackets. With regards to the observation geometry, we choose $\phi=\pi / 2$ which restricts us to the $y>0$ region of the $y-z$ plane, with

$$
\begin{aligned}
& \hat{\mathbf{r}}=\sin \theta \hat{\mathbf{y}}+\cos \theta \hat{\mathbf{z}} \\
& \hat{\boldsymbol{\theta}}=\cos \theta \hat{\mathbf{y}}-\sin \theta \hat{\mathbf{z}} \\
& \hat{\boldsymbol{\phi}}=-\hat{\mathbf{x}}
\end{aligned}
$$

Note that this choice does not limit the generality of the results below.

Writing down the rotationally averaged Stokes parameters $\left\langle s_{\xi}\right\rangle$ of the scattered light in terms of the Stokes parameters $S_{\xi}$ of the incident light involves calculating the rotational averages of (25)-(32) and using (33)-(41). Some of the terms in the second-order correction, embodied here by the $\left\langle s_{\xi}^{(2)}\right\rangle$, have the same dependences on the $S_{\xi}$ and the scattering angle $\theta$ as terms in the zerothorder theory, embodied here by the $\left\langle s_{\xi}^{(0)}\right\rangle$. We therefore consider the $\left\langle s_{\xi}^{(0)}\right\rangle$ and the $\left\langle s_{\xi}^{(2)}\right\rangle$ simultaneously. The general results are listed in appendix C. For the special case of Rayleigh scattering of far-off-resonance light by a time-reversible scatterer (see appendix A 3), they reduce to the following forms:

$$
\begin{aligned}
\left\langle s_{0}^{(0)}\right\rangle+\left\langle s_{0}^{(2)}\right\rangle & =K\left[S_{0}\left(\mathrm{~A}+\cos \theta \mathrm{B}^{\prime}+\cos ^{2} \theta \mathrm{C}+\cos ^{3} \theta \mathrm{D}^{\prime \prime}\right)\right. \\
& \left.+S_{1} \sin ^{2} \theta\left(\mathrm{E}+\cos \theta \mathrm{F}^{\prime \prime}\right)\right] \\
\left\langle s_{1}^{(0)}\right\rangle+\left\langle s_{1}^{(2)}\right\rangle & =K\left[S_{1}\left(\mathrm{G}+\cos \theta \mathrm{H}^{\prime}+\cos ^{2} \theta \mathrm{I}+\cos ^{3} \theta \mathrm{J}^{\prime \prime}\right)\right. \\
& \left.+S_{0} \sin ^{2} \theta\left(\mathrm{K}+\cos \theta \mathrm{L}^{\prime \prime}\right)\right] \\
\left\langle s_{2}^{(0)}\right\rangle+\left\langle s_{2}^{(2)}\right\rangle & =K S_{2}\left(\mathrm{M}^{\prime}+\cos \theta \mathrm{N}+\cos ^{2} \theta \mathrm{O}^{\prime}\right) \\
\left\langle s_{3}^{(0)}\right\rangle+\left\langle s_{3}^{(2)}\right\rangle & =K S_{3}\left(\mathrm{P}^{\prime}+\cos \theta \mathrm{Q}+\cos ^{2} \theta \mathrm{R}^{\prime}\right)
\end{aligned}
$$

Explicit expressions for the coefficients $\mathrm{A}, \ldots, \mathrm{R}^{\prime}$ are listed in appendix D. Note that $\left\langle s_{0}^{(0)}\right\rangle+\left\langle s_{0}^{(2)}\right\rangle$ and $\left\langle s_{1}^{(0)}\right\rangle+\left\langle s_{1}^{(2)}\right\rangle$ are independent of $S_{2}$ and $S_{3}$ and that $\left\langle s_{2}^{(0)}\right\rangle+\left\langle s_{2}^{(2)}\right\rangle$ and $\left\langle s_{3}^{(0)}\right\rangle+\left\langle s_{3}^{(2)}\right\rangle$ are independent of $S_{0}$ and $S_{1}$. Furthemore for $\theta=0$ and $\theta=\pi$ we obtain $\left\langle s_{0}^{(0)}\right\rangle+\left\langle s_{0}^{(2)}\right\rangle \propto S_{0},\left\langle s_{1}^{(0)}\right\rangle+$ $\left\langle s_{1}^{(2)}\right\rangle \propto S_{1},\left\langle s_{2}^{(0)}\right\rangle+\left\langle s_{2}^{(2)}\right\rangle \propto S_{2}$ and $\left\langle s_{3}^{(0)}\right\rangle+\left\langle s_{3}^{(2)}\right\rangle \propto S_{3}$.

The coefficients $\mathrm{A}, \ldots, \mathrm{R}^{\prime}$ can be grouped into three types by their dependence on different subsets of the property tensors. The unprimed coefficients (A, C, E, G, I, $\mathrm{K}, \mathrm{N}$ and $\mathrm{Q}$ ) each have contributions of the following types: ' $\alpha-\alpha$ ', ' $A-A$ ', ' $G-A$ ', ' $G-G$ ', ' $\alpha-B$ ' and ' $\alpha-D^{m}$ '. The singly-primed coefficients $\left(\mathrm{B}^{\prime}, \mathrm{H}^{\prime}, \mathrm{M}^{\prime}, \mathrm{O}^{\prime}, \mathrm{P}^{\prime}\right.$ and $\mathrm{R}^{\prime}$ ) each have contributions of the following types: ' $A-A$ ', ' $G-A$ ', ' $G-G$ ', ' $\alpha-C$ ', ' $\alpha-D$ ' and ' $\alpha-\chi$ '. Note that there are no contributions of the ' $\alpha-\alpha$ ' type here: the singly primed coefficients are of pure second-order character. Finally, the doubly-primed coefficients $\left(D^{\prime \prime}, \mathrm{F}^{\prime \prime}, \mathrm{J}^{\prime \prime}\right.$ and $\mathrm{L}^{\prime \prime}$ ) each have contributions of the types ' $A-A$ ' and ' $\alpha-C$ '. Note that the doubly primed coefficients are also of pure second-order character.
The first-order correction, embodied here by the $\left\langle s_{\xi}^{(1)}\right\rangle$, is of a rather different character to the zeroth-order theory and its second-order correction, embodied here by the $\left\langle s_{\xi}^{(0)}\right\rangle$ and the $\left\langle s_{\xi}^{(2)}\right\rangle$. In particular, it has different, optically active dependencies upon the Stokes parameters $S_{\xi}$ of the incident light. In contrast, each of the coefficients $\mathrm{A}, \ldots, \mathrm{R}^{\prime}$ is unchanged when the scatterer is inverted through the spatial origin. That is to say, the rotationally averaged zeroth-order theory and its secondorder correction are independent of the scatterer's chirality: they don't discriminate between left and right. For a particularly clear discussion of the $\left\langle s_{\xi}^{(1)}\right\rangle$, see [41].

\section{OUTLOOK}

In this paper we have focussed upon a single scatterer. Our results are most relevant to elastic light scattering in samples for which the scatterers can be regarded as independent and in which multiple scattering is not important (a rarefied medium such as an ideal gas being the prototypical example) and to inelastic light scattering at essentially all sample densities, again provided that multiple scattering is not important $[1,2]$. It remains to incorporate our results into more realistic, sample-specific theories, where the motions of the scatterers, local field corrections and other subtleties are taken into account. This is especially important for small scatterers and / or long wavelengths, as the second-order correction will be especially small in such cases.

An obvious next step is to explore potential applications. A group-theoretical analysis of the coefficients $A, \ldots, R^{\prime}$ could prove useful here, as it might facilitate a better understanding of their dependence upon the shape and other properties of a scatterer (we already know that $A, \ldots, R^{\prime}$ are independent of a scatterer's chirality, for example). One might hope to find a measurable combination of $A, \ldots, R^{\prime}$ that distinguishes between chirality and achirality to directly probe the chirality of scatterers in racemic mixtures, or a combination that is uniquely sensitive to icosahedral scatterers for the purposes of virus detection, for example. It is also necessary to identify experimental arrangements optimised towards the secondorder correction, as the signatures of interest will invariably be small. Spatially structured light could prove useful here. Consider the rotational average of our results for a scatterer located in the node of a linearly polarised standing wave, for example: there is no scattering to zeroth-order (as $\mathbf{E}=0$ ) or first-order (as the illuminating light is achiral $[37,38])$ and the second-order correction describes the scattered light to leading order.

It is natural, perhaps, to enquire about the third-order correction to the zeroth-order Rayleigh / Raman theory, although a basic estimate reveals that this will be smaller still than the second-order correction by a factor of $\sim k_{0} d$. It seems that some progress in this direction has already been made, however: we believe that the novel diamag- 
netic light scattering described in [11] can be regarded as part of an anticipated ' $\chi-\chi$ ' contribution to the thirdorder correction.

\section{ACKNOWLEDGMENTS}

Robert P. Cameron gratefully acknowledges support from the Engineering and Physical Sciences Research Council (EP/M004694/1) and the Leverhulme Trust (RPG-2017-048). Neel Mackinnon thanks the Engineering and Physical Sciences Research Council for the award of a studentship.

\section{Appendix A: Explicit quantum-mechanical expressions}

In this appendix we give explicit quantum-mechanical expressions for multipole moments and property tensors. We also show how the latter reduce under certain special circumstances.

Let us suppose that the scatterer is a molecule in vacuum: an electrically neutral collection of electrons and nuclei, bound together by electromagnetic interactions. We treat the $k$ th particle (electron or nucleus) as a point-like object of rest mass $m_{k}$, mean position $\hat{\mathbf{r}}_{k}=\mathbf{r}_{k}$, canonical momentum $\hat{\mathbf{p}}_{k}=-\mathrm{i} \hbar \nabla_{k}$, electric charge $q_{k}$ and magnetic-dipole moment $\hat{\mathbf{m}}_{k}=\gamma_{k} \hat{\mathbf{s}}_{k}$, with $\gamma_{k}$ the gyromagnetic ratio and $\hat{\mathbf{s}}_{k}=\hbar \boldsymbol{\sigma}_{k} / 2$ the mean spin, where $\boldsymbol{\sigma}_{k}$ is a pseudovector of Pauli matrices. We indicate dependencies upon position $\mathbf{r}=x \hat{\mathbf{x}}+y \hat{\mathbf{y}}+z \hat{\mathbf{z}}$ but refrain from indicating dependencies on time $t$, for the sake of notational simplicity.

Working to order $1 / c^{0}$, the molecular Hamiltonian is

$$
\hat{H}=\hat{H}_{0}+\hat{V}
$$

with

$$
\hat{H}_{0}=\sum_{k} \frac{\hat{\mathbf{p}}_{k}^{2}}{2 m_{k}}+\sum_{k} \sum_{k^{\prime} \neq k} \frac{q_{k} q_{k^{\prime}}}{8 \pi \epsilon_{0}\left|\hat{\mathbf{r}}_{k}-\hat{\mathbf{r}}_{k^{\prime}}\right|}
$$

the unperturbed molecular Hamiltonian and

$$
\begin{aligned}
\hat{V} & =\sum_{k} q_{k} \Phi\left(\hat{\mathbf{r}}_{k}\right) \\
& -\sum_{k} \frac{q_{k}}{2 m_{k}}\left[\hat{\mathbf{p}}_{k} \cdot \mathbf{A}\left(\hat{\mathbf{r}}_{k}\right)+\mathbf{A}\left(\hat{\mathbf{r}}_{k}\right) \cdot \hat{\mathbf{p}}_{k}\right] \\
& -\sum_{k} \hat{\mathbf{m}}_{k} \cdot \mathbf{B}\left(\hat{\mathbf{r}}_{k}\right)+\sum_{k} \frac{q_{k}^{2}}{2 m_{k}} \mathbf{A}^{2}\left(\hat{\mathbf{r}}_{k}\right)
\end{aligned}
$$

the interaction Hamiltonian, where $\Phi(\mathbf{r})$ and $\mathbf{A}(\mathbf{r})$ are the scalar and magnetic-vector potentials of the illuminating light, the electric and magnetic fields of which follow as

$$
\begin{aligned}
& \mathbf{E}(\mathbf{r})=-\nabla \Phi(\mathbf{r})-\dot{\mathbf{A}}(\mathbf{r}) \\
& \mathbf{B}(\mathbf{r})=\nabla \times \mathbf{A}(\mathbf{r}) .
\end{aligned}
$$

For a detailed discussion of the relativistic properties of a molecule to order $1 / c^{2}$, see [42]. We imagine that the energy spectrum of the molecule in isolation is known:

$$
\hat{H}_{0}|s\rangle=\hbar \omega_{s}|s\rangle
$$

with $|s\rangle$ the unperturbed energy eigenstates and $\hbar \omega_{s}$ the associated energy eigenvalues.

Working to order $\lambda^{2}$, we now choose the following potentials [28, 34]:

$$
\begin{aligned}
\Phi(\mathbf{r}) & \approx \lambda^{-1} \Phi-\lambda^{0} r_{a} E_{a}-\lambda^{1} \frac{1}{2} r_{a} r_{b} \partial_{b} E_{a} \\
& -\lambda^{2} \frac{1}{6} r_{a} r_{b} r_{c} \partial_{c} \partial_{b} E_{a} \\
A_{a}(\mathbf{r}) & \approx-\lambda^{1} \frac{1}{2} \epsilon_{a b c} r_{b} B_{c}-\lambda^{2} \frac{1}{3} \epsilon_{a b c} r_{b} r_{d} \partial_{d} B_{c},
\end{aligned}
$$

with $\Phi=\Phi(\mathbf{r}=0)$, for example. Substituting (A7) and (A8) into (A3) yields the following multipolar expansion about the spatial origin:

$$
\begin{aligned}
\hat{V} & \approx-\lambda^{0} \hat{\mu}_{a} E_{a}-\lambda^{1} \hat{m}_{a} B_{a}-\lambda^{1} \frac{1}{3} \hat{\Theta}_{a b} \partial_{b} E_{a} \\
& -\lambda^{2} \frac{1}{2} \hat{m}_{a b} \partial_{b} B_{a}-\lambda^{2} \frac{1}{6} \hat{Q}_{a b c} \partial_{c} \partial_{b} E_{a},
\end{aligned}
$$

with the multipole moments $\hat{\mu}_{a}, \hat{m}_{a}, \hat{\Theta}_{a b}, \hat{m}_{a b}$ and $\hat{Q}_{a b c}$ as defined below. We have neglected nonlinear terms in (A9), as our interest is in linear light scattering.

\section{Multipole moments}

We define the (components of the) electric-dipole moment $\hat{\mu}_{a}$, the canonical magnetic-dipole moment $\hat{m}_{a}$, the mechanical magnetic-dipole moment $\hat{M}_{a}$, the symmetric and traceless electric-quadrupole moment $\hat{\Theta}_{a b}$, the canonical magnetic-quadrupole moment $\hat{m}_{a b}$, the mechanical magnetic-quadrupole moment $\hat{M}_{a b}$ and the electric-octupole moment $\hat{Q}_{a b c}$ of the molecule about the 
spatial origin as follows [2, 34, 43]:

$$
\begin{aligned}
\hat{\mu}_{a} & =\sum_{k} q_{k} \hat{r}_{k a}, \\
\hat{m}_{a} & =\sum_{k} \frac{q_{k}}{2 m_{k}}\left(\epsilon_{a b c} \hat{r}_{k b} \hat{p}_{k c}+\frac{2 m_{k} \gamma_{k}}{q_{k}} \hat{s}_{k a}\right), \\
\hat{M}_{a} & =\sum_{k} \frac{q_{k}}{2}\left(\epsilon_{a b c} \hat{r}_{k b} \hat{v}_{k c}+\frac{2 \gamma_{k}}{q_{k}} \hat{s}_{k a}\right), \\
\hat{\Theta}_{a b} & =\sum_{k} \frac{q_{k}}{2}\left(3 \hat{r}_{k a} \hat{r}_{k b}-\delta_{a b} \hat{r}_{k c} \hat{r}_{k c}\right), \\
\hat{m}_{a b} & =\sum_{k} \frac{q_{k}}{m_{k}}\left[\hat{r}_{k b}\left(\frac{1}{3} \epsilon_{a c d} \hat{r}_{k c} \hat{p}_{k d}+\frac{m_{k} \gamma_{k}}{q_{k}} \hat{s}_{k a}\right)\right. \\
& \left.+\left(\frac{1}{3} \epsilon_{a c d} \hat{r}_{k c} \hat{p}_{k d}+\frac{m_{k} \gamma_{k}}{q_{k}} \hat{s}_{k a}\right) \hat{r}_{k b}\right], \\
\hat{M}_{a b} & =\sum_{k} q_{k}\left[\hat{r}_{k b}\left(\frac{1}{3} \epsilon_{a c d} \hat{r}_{k c} \hat{v}_{k d}+\frac{\gamma_{k}}{q_{k}} \hat{s}_{k a}\right)\right. \\
& \left.+\left(\frac{1}{3} \epsilon_{a c d} \hat{r}_{k c} \hat{v}_{k d}+\frac{\gamma_{k}}{q_{k}} \hat{s}_{k a}\right) \hat{r}_{k b}\right] \\
\hat{Q}_{a b c} & =\sum_{k} q_{k} \hat{r}_{k a} \hat{r}_{k b} \hat{r}_{k c},
\end{aligned}
$$

with

$$
\hat{\mathbf{v}}_{k}=\frac{1}{m_{k}}\left[\hat{\mathbf{p}}_{k}-q_{k} \mathbf{A}\left(\hat{\mathbf{r}}_{k}\right)\right]
$$

the velocity of the $k$ th particle. Working to order $\lambda^{2}$, we use (A8) to deduce that

$$
\begin{aligned}
\lambda^{1} \hat{M}_{a} & =\lambda^{1} \hat{m}_{a}-\lambda^{1} \sum_{k} \frac{q_{k}^{2}}{2 m_{k}} \epsilon_{a b c} \hat{r}_{k b} A_{c}\left(\hat{\mathbf{r}}_{k}\right) \\
& \approx \lambda^{1} \hat{m}_{a}+\lambda^{2} \hat{\chi}_{a b}^{(d)} B_{b} \\
\lambda^{2} \hat{M}_{a b} & =\lambda^{2} \hat{m}_{a b}-\lambda^{2} \sum_{k} \frac{2 q_{k}^{2}}{3 m_{k}} \epsilon_{a c d} \hat{r}_{k b} \hat{r}_{k c} A_{d}\left(\hat{\mathbf{r}}_{k}\right) \\
& \approx \lambda^{2} \hat{m}_{a b},
\end{aligned}
$$

with

$$
\hat{\chi}_{a b}^{(d)}=\sum_{k} \frac{q_{k}^{2}}{4 m_{k}}\left(\hat{r}_{k a} \hat{r}_{k b}-\delta_{a b} \hat{r}_{k c} \hat{r}_{k c}\right)
$$

the diamagnetic susceptibility of the molecule about the spatial origin. It will prove useful in appendix B to recognise, in addition to (7)-(11), the following:

$$
\begin{aligned}
\lambda^{1} \tilde{N}_{a} & \approx \lambda^{1} \tilde{\mathscr{G}}_{a b}^{(n)} \tilde{E}_{b}+\lambda^{2} \frac{1}{3} \tilde{D}_{a, b c}^{(n)} \partial_{c} \tilde{E}_{b} \\
& +\lambda^{2} \tilde{\chi}_{a b}^{(n)} \tilde{B}_{b} \\
\lambda^{1} \tilde{Q}_{a b} & \approx \lambda^{1} \tilde{\mathscr{A}}_{c, a b}^{(Q)} \tilde{E}_{c}+\lambda^{2} \tilde{C}_{a b, c d}^{(Q)} \partial_{d} \tilde{E}_{c} \\
& +\lambda^{2} \tilde{\mathscr{D}}_{c, a b}^{(Q)} \tilde{B}_{c},
\end{aligned}
$$

with the property tensors $\tilde{\mathscr{G}}_{a b}^{(n)}, \tilde{D}_{a, b c}^{(n)}, \tilde{\chi}_{a b}^{(n)}, \tilde{\mathscr{A}}_{a, b c}^{(Q)}, \tilde{C}_{a b, c d}^{(Q)}$ and $\tilde{\mathscr{D}}_{a, b c}^{(Q)}$ defined identically to the property tensors $\tilde{\mathscr{G}}_{a b}$,
$\tilde{D}_{a, b c}, \tilde{\chi}_{a b}, \tilde{\mathscr{A}}_{a, b c}, \tilde{C}_{a b, c d}$ and $\tilde{\mathscr{D}}_{a, b c}$ but with $\hat{m}_{a}$ replaced by the canonical orbital magnetic-dipole moment $\hat{n}_{a}$ of the molecule about the spatial origin in the former three and $\hat{\Theta}_{a b}$ replaced by the symmetric but not traceless electric-quadrupole moment $\hat{Q}_{a b}$ of the molecule about the spatial origin in the latter three, where

$$
\begin{aligned}
\hat{n}_{a} & =\sum_{k} \frac{q_{k}}{2 m_{k}} \epsilon_{a b c} \hat{r}_{k b} \hat{p}_{k c} \\
\hat{Q}_{a b} & =\sum_{k} q_{k} \hat{r}_{k a} \hat{r}_{k b} .
\end{aligned}
$$

Working to order $\lambda^{2}$, we can again use (A8) to relate the mechanical orbital magnetic-dipole moment $\hat{N}_{a}$ of the molecule about the spatial origin to $\hat{n}_{a}$ :

$$
\begin{aligned}
\lambda^{1} \hat{N}_{a} & =\lambda^{1} \sum_{k} \frac{q_{k}}{2} \epsilon_{a b c} \hat{r}_{k b} \hat{v}_{k c} \\
& =\lambda^{1} \hat{n}_{a}-\lambda^{1} \sum_{k} \frac{q_{k}^{2}}{2 m_{k}} \epsilon_{a b c} \hat{r}_{k b} A_{c}\left(\hat{\mathbf{r}}_{k}\right) \\
& \approx \lambda^{1} \hat{n}_{a}+\lambda^{2} \hat{\chi}_{a b}^{(d)} B_{b},
\end{aligned}
$$

which is distinct from (A18).

\section{Property tensors}

A simple quantum-mechanical treatment akin to that given in $[2,34,43]$ gives the following explicit forms for 
the molecule's property tensors:

$$
\begin{aligned}
& \tilde{\alpha}_{a b}=\frac{1}{\hbar} \sum_{s}\left(\frac{\mu_{a}^{f s} \mu_{b}^{s i}}{\tilde{\omega}_{s i}-\omega_{0}}+\frac{\mu_{b}^{f s} \mu_{a}^{s i}}{\tilde{\omega}_{s f}^{*}+\omega_{0}}\right), \\
& \tilde{A}_{a, b c}=\frac{1}{\hbar} \sum_{s}\left(\frac{\mu_{a}^{f s} \Theta_{b c}^{s i}}{\tilde{\omega}_{s i}-\omega_{0}}+\frac{\Theta_{b c}^{f s} \mu_{a}^{s i}}{\tilde{\omega}_{s f}^{*}+\omega_{0}}\right) \text {, } \\
& \tilde{\mathscr{A}}_{a, b c}=\frac{1}{\hbar} \sum_{s}\left(\frac{\Theta_{b c}^{f s} \mu_{a}^{s i}}{\tilde{\omega}_{s i}-\omega_{0}}+\frac{\mu_{a}^{f s} \Theta_{b c}^{s i}}{\tilde{\omega}_{s f}^{*}+\omega_{0}}\right), \\
& \tilde{G}_{a b}=\frac{1}{\hbar} \sum_{s}\left(\frac{\mu_{a}^{f s} m_{b}^{s i}}{\tilde{\omega}_{s i}-\omega_{0}}+\frac{m_{b}^{f s} \mu_{a}^{s i}}{\tilde{\omega}_{s f}^{*}+\omega_{0}}\right), \\
& \tilde{\mathscr{G}}_{a b}=\frac{1}{\hbar} \sum_{s}\left(\frac{m_{a}^{f s} \mu_{b}^{s i}}{\tilde{\omega}_{s i}-\omega_{0}}+\frac{\mu_{b}^{f s} m_{a}^{s i}}{\tilde{\omega}_{s f}^{*}+\omega_{0}}\right), \\
& \tilde{B}_{a, b c d}=\frac{1}{\hbar} \sum_{s}\left(\frac{\mu_{a}^{f s} Q_{b c d}^{s i}}{\tilde{\omega}_{s i}-\omega_{0}}+\frac{Q_{b c d}^{f s} \mu_{a}^{s i}}{\tilde{\omega}_{s f}^{*}+\omega_{0}}\right) \text {, } \\
& \tilde{\mathscr{B}}_{a, b c d}=\frac{1}{\hbar} \sum_{s}\left(\frac{Q_{b c d}^{f s} \mu_{a}^{s i}}{\tilde{\omega}_{s i}-\omega_{0}}+\frac{\mu_{a}^{f s} Q_{b c d}^{s i}}{\tilde{\omega}_{s f}^{*}+\omega_{0}}\right) \text {, } \\
& \tilde{C}_{a b, c d}=\frac{1}{3 \hbar} \sum_{s}\left(\frac{\Theta_{a b}^{f s} \Theta_{c d}^{s i}}{\tilde{\omega}_{s i}-\omega_{0}}+\frac{\Theta_{c d}^{f s} \Theta_{a b}^{s i}}{\tilde{\omega}_{s f}^{*}+\omega_{0}}\right), \\
& \tilde{D}_{a, b c}=\frac{1}{\hbar} \sum_{s}\left(\frac{m_{a}^{f s} \Theta_{b c}^{s i}}{\tilde{\omega}_{s i}-\omega_{0}}+\frac{\Theta_{b c}^{f s} m_{a}^{s i}}{\tilde{\omega}_{s f}^{*}+\omega_{0}}\right), \\
& \tilde{\mathscr{D}}_{a, b c}=\frac{1}{\hbar} \sum_{s}\left(\frac{\Theta_{b c}^{f s} m_{a}^{s i}}{\tilde{\omega}_{s i}-\omega_{0}}+\frac{m_{a}^{f s} \Theta_{b c}^{s i}}{\tilde{\omega}_{s f}^{*}+\omega_{0}}\right), \\
& \tilde{D}_{a, b c}^{(m)}=\frac{1}{\hbar} \sum_{s}\left(\frac{\mu_{a}^{f s} m_{b c}^{s i}}{\tilde{\omega}_{s i}-\omega_{0}}+\frac{m_{b c}^{f s} \mu_{a}^{s i}}{\tilde{\omega}_{s f}^{*}+\omega_{0}}\right), \\
& \tilde{\mathscr{D}}_{c, a b}^{(m)}=\frac{1}{\hbar} \sum_{s}\left(\frac{m_{a b}^{f s} \mu_{c}^{s i}}{\tilde{\omega}_{s i}-\omega_{0}}+\frac{\mu_{c}^{f s} m_{a b}^{s i}}{\tilde{\omega}_{s f}^{*}+\omega_{0}}\right) \\
& \tilde{\chi}_{a b}=\left\langle f\left|\hat{\chi}_{a b}^{(d)}\right| i\right\rangle \\
& +\frac{1}{\hbar} \sum_{s}\left(\frac{m_{a}^{f s} m_{b}^{s i}}{\tilde{\omega}_{s i}-\omega_{0}}+\frac{m_{b}^{f s} m_{a}^{s i}}{\tilde{\omega}_{s f}^{*}+\omega_{0}}\right),
\end{aligned}
$$

with

$$
\tilde{\omega}_{f i}=\omega_{f i}-\frac{1}{2} \mathrm{i} \Gamma_{f i}
$$

a complex transition angular frequency, where $\mu_{a}^{s i}=$ $\left\langle s\left|\hat{\mu}_{a}\right| i\right\rangle$, for example. We have dropped explicit state exclusions from the summations [2] and refrained from using tildes for potentially complex matrix elements, for the sake of notational simplicity.

\section{Reduced property tensors}

For the special case of Rayleigh scattering of far-offresonance light by a time-reversible scatterer $\omega=\omega_{0}$, and we approximate the property tensors by the following forms:

$$
\begin{aligned}
\tilde{\alpha}_{a b} & =\alpha_{a b}, \\
\tilde{A}_{a, b c} & =A_{a, b c}, \\
\tilde{\mathscr{A}}_{a, b c} & =A_{a, b c}, \\
\tilde{G}_{a b} & =-\mathrm{i} G_{a b}^{\prime}, \\
\tilde{\mathscr{G}}_{a b} & =\mathrm{i} G_{b a}^{\prime}, \\
\tilde{C}_{a b, c d} & =C_{a b, c d}, \\
\tilde{B}_{a, b c d} & =B_{a, b c d}, \\
\tilde{\mathscr{B}}_{a, b c d} & =B_{a, b c d}, \\
\tilde{D}_{a, b c} & =-\mathrm{i} D_{a, b c}^{\prime}, \\
\tilde{\mathscr{D}}_{a, b c} & =\mathrm{i} D_{a, b c}^{\prime}, \\
\tilde{D}_{a, b c}^{(m)} & =-\mathrm{i} D_{a, b c}^{(m)^{\prime}}, \\
\tilde{\mathscr{D}}_{a, b c}^{(m)} & =\mathrm{i} D_{a, b c}^{(m)^{\prime}} \\
\tilde{\chi}_{a b} & =\chi_{a b},
\end{aligned}
$$

with, for a molecule in particular,

$$
\begin{aligned}
\alpha_{a b} & =\frac{2}{\hbar} \sum_{s} \frac{\omega_{s i}}{\omega_{s i}^{2}-\omega_{0}^{2}} \mathfrak{R}\left(\mu_{a}^{i s} \mu_{b}^{s i}\right), \\
A_{a, b c} & =\frac{2}{\hbar} \sum_{s} \frac{\omega_{s i}}{\omega_{s i}^{2}-\omega_{0}^{2}} \mathfrak{R}\left(\mu_{a}^{i s} \Theta_{b c}^{s i}\right) \\
G_{a b}^{\prime} & =-\frac{2}{\hbar} \sum_{s} \frac{\omega_{0}}{\omega_{s i}^{2}-\omega_{0}^{2}} \Im\left(\mu_{a}^{i s} m_{b}^{s i}\right), \\
B_{a, b c d} & =\frac{2}{\hbar} \sum_{s} \frac{\omega_{s i}}{\omega_{s i}^{2}-\omega_{0}^{2}} \mathfrak{R}\left(\mu_{a}^{i s} Q_{b c d}^{s i}\right), \\
C_{a b, c d} & =\frac{2}{3 \hbar} \sum_{s} \frac{\omega_{s i}}{\omega_{s i}^{2}-\omega_{0}^{2}} \mathfrak{R}\left(\Theta_{a b}^{i s} \Theta_{b c}^{s i}\right), \\
D_{a, b c}^{\prime} & =-\frac{2}{\hbar} \sum_{s} \frac{\omega_{0}}{\omega_{s i}^{2}-\omega_{0}^{2}} \Im\left(m_{a}^{i s} \Theta_{b c}^{s i}\right), \\
D_{a, b c}^{(m)^{\prime}} & =-\frac{2}{\hbar} \sum_{s} \frac{\omega_{0}}{\omega_{s i}^{2}-\omega_{0}^{2}} \Im\left(\mu_{a}^{i s} m_{b c}^{s i}\right) \\
\chi_{a b} & =\left\langle i\left|\hat{\chi}_{a b}^{(d)}\right| i\right\rangle+\frac{2}{\hbar} \sum_{s} \frac{\omega_{s i}}{\omega_{s i}^{2}-\omega_{0}^{2}} \mathfrak{R}\left(m_{a}^{i s} m_{b}^{s i}\right) .
\end{aligned}
$$

Again, we have dropped explicit state exclusions from the summations [2] and refrained from using tildes for potentially complex matrix elements.

\section{Reduced property tensors for a scatterer with spherical symmetry}

For a scatterer with spherical symmetry, the reduced property tensors described above reduce further to the 
following forms:

$$
\begin{aligned}
\alpha_{a b} & =\delta_{a b} \alpha, \\
A_{a, b c} & =0, \\
G_{a b}^{\prime} & =\delta_{a b} G^{\prime}, \\
B_{a, b c d} & =\left(\delta_{a b} \delta_{c d}+\delta_{a c} \delta_{b d}+\delta_{a d} \delta_{b c}\right) B, \\
C_{a b, c d} & =\left[2 \delta_{a b} \delta_{c d}-3\left(\delta_{a c} \delta_{b d}+\delta_{a d} \delta_{b c}\right)\right] C, \\
D_{a, b c}^{\prime} & =0, \\
D_{a, b c}^{(m)^{\prime}} & =\epsilon_{a b c} D^{\prime(m)} \\
\chi_{a b} & =\delta_{a b} \chi,
\end{aligned}
$$

with the scalars $\alpha, G^{\prime}, B, C, D^{\prime(m)}$ and $\chi$ dependent upon the precise nature of the scatterer, of course. Deviations away from the forms seen in (A61)-(A68) encode information about the shape of the scatterer.

\section{Appendix B: Origin independence}

In this appendix we show that our physical predictions are independent of our choice of origin for multipolar expansions, as they should be. The calculations here are rather intricate and we focus our attention, therefore, upon Rayleigh scattering of far-off-resonance light by a time-reversible scatterer (see appendix A 3).

Elsewhere in this paper we have chosen the origin of our multipolar expansions to coincide with the spatial origin. Here we choose a different origin for our multipolar expansions, located at position a (sufficiently close to the spatial origin to ensure converging multipolar expansions). We use a prime to indicate that a quantity is defined relative to $\mathbf{a}$. This should cause no confusion, except perhaps in the case of the reduced property tensors $G_{a b}^{\prime}, D_{a, b c}^{\prime}$ and $D_{a, b c}^{(m)^{\prime}}$, which are defined relative to the spatial origin and already have a prime (as per the conventional notation): we denote the corresponding quantities defined relative to a as $\left(G_{a b}^{\prime}\right)^{\prime},\left(D_{a, b c}^{\prime}\right)^{\prime}$ and $\left(D_{a, b c}^{(m)^{\prime}}\right)^{\prime}$.

In place of (A7) and (A8) we choose

$$
\begin{aligned}
& \Phi^{\prime}(\mathbf{r}) \approx \lambda^{-1} \Phi^{\prime}-\lambda^{0} r_{a}^{\prime} E_{a}^{\prime}-\lambda^{1} \frac{1}{2} r_{a}^{\prime} r_{b}^{\prime} \partial_{b} E_{a}^{\prime} \\
&-\lambda^{2} \frac{1}{6} r_{a}^{\prime} r_{b}^{\prime} r_{c}^{\prime} \partial_{c} \partial_{b} E_{a}^{\prime} \\
& A_{a}^{\prime}(\mathbf{r}) \approx-\lambda^{1} \frac{1}{2} \epsilon_{a b c} r_{b}^{\prime} B_{c}^{\prime}-\lambda^{2} \frac{1}{3} \epsilon_{a b c} r_{b}^{\prime} r_{d}^{\prime} \partial_{d} B_{c}^{\prime},
\end{aligned}
$$

with $\mathbf{r}^{\prime}=\mathbf{r}-\mathbf{a}$ and $\Phi^{\prime}=\Phi^{\prime}(\mathbf{r}=\mathbf{a})$, for example. Note that (B1) and (B2) differ from (A7) and (A8) by a gauge transformation. In place of (A9) we obtain the following multipolar expansion about $\mathbf{a}$ :

$$
\begin{aligned}
\hat{V}^{\prime} & \approx-\lambda^{0} \hat{\mu}_{a}^{\prime} E_{a}^{\prime}-\lambda^{1} \hat{m}_{a}^{\prime} B_{a}^{\prime}-\lambda^{1} \frac{1}{3} \hat{\Theta}_{a b}^{\prime} \partial_{b} E_{a}^{\prime} \\
& -\lambda^{2} \frac{1}{2} \hat{m}_{a b}^{\prime} \partial_{b} B_{a}^{\prime}-\lambda^{2} \frac{1}{6} \hat{Q}_{a b c}^{\prime} \partial_{c} \partial_{b} E_{a}^{\prime},
\end{aligned}
$$

with the primed multipole moments $\hat{\mu}_{a}^{\prime}, \hat{m}_{a}^{\prime}, \hat{\Theta}_{a b}^{\prime}, \hat{m}_{a b}^{\prime}$ and $\hat{Q}_{a b c}^{\prime}$ defined identically to the unprimed multipole moments $\hat{\mu}_{a}, \hat{m}_{a}, \hat{\Theta}_{a b}, \hat{m}_{a b}$ and $\hat{Q}_{a b c}$ but with the position $\hat{\mathbf{r}}_{k}$ translated as $\hat{\mathbf{r}}_{k}^{\prime}=\hat{\mathbf{r}}_{k}-\mathbf{a}$. Thus

$$
\begin{aligned}
\hat{\mu}_{a}^{\prime} & =\sum_{k} q_{k} \hat{r}_{k a}^{\prime} \\
\hat{m}_{a}^{\prime} & =\sum_{k} \frac{q_{k}}{2 m_{k}}\left(\epsilon_{a b c} \hat{r}_{k b}^{\prime} \hat{p}_{k c}+\frac{2 m_{k} \gamma_{k}}{q_{k}} \hat{s}_{k a}\right),
\end{aligned}
$$

for example. It follows that

$$
\begin{aligned}
\hat{\mu}_{a}^{\prime} & =\hat{\mu}_{a} \\
\hat{m}_{a}^{\prime} & =\hat{m}_{a}-\frac{1}{2} \epsilon_{a b c} a_{b} \frac{\mathrm{i}}{\hbar}\left[\hat{H}_{0}, \hat{\mu}_{c}\right], \\
\hat{\Theta}_{a b}^{\prime} & =\hat{\Theta}_{a b}-\frac{3}{2} a_{a} \hat{\mu}_{b}-\frac{3}{2} a_{b} \hat{\mu}_{a}+\delta_{a b} a_{c} \hat{\mu}_{c} \\
\hat{m}_{a b}^{\prime} & =\hat{m}_{a b}+\frac{2}{3} \epsilon_{a c d} a_{b} a_{c} \frac{\mathrm{i}}{\hbar}\left[\hat{H}_{0}, \hat{\mu}_{d}\right] \\
& -2 a_{b} \hat{m}_{a}-\frac{1}{3} \epsilon_{a c d} a_{c} \frac{\mathrm{i}}{\hbar}\left[\hat{H}_{0}, \hat{Q}_{b d}\right] \\
& +\frac{2}{3} \delta_{a b} a_{c} \hat{n}_{c} \\
\hat{Q}_{a b c}^{\prime} & =\hat{Q}_{a b c}+a_{a} a_{b} \hat{\mu}_{c}+a_{a} a_{c} \hat{\mu}_{b}+a_{b} a_{c} \hat{\mu}_{a} \\
& -a_{a} \hat{Q}_{b c}-a_{b} \hat{Q}_{a c}-a_{c} \hat{Q}_{a b} .
\end{aligned}
$$

Note that $\hat{Q}_{a b}$ and $\hat{n}_{a}$ have emerged naturally here, as anticipated in appendix A 1 . Working to order $\lambda^{2}$, we use (B2) to deduce that the primed mechanical magneticdipole moment of the molecule is

$$
\begin{aligned}
\lambda^{1} \hat{M}_{a}^{\prime} & =\lambda^{1} \sum_{k} \frac{q_{k}}{2} \epsilon_{a b c} \hat{r}_{k b}^{\prime} \hat{v}_{k c}^{\prime} \\
& =\lambda^{1} \hat{m}_{a}^{\prime}-\lambda^{1} \sum_{k} \frac{q_{k}^{2}}{2 m_{k}} \epsilon_{a b c} \hat{r}_{k b}^{\prime} A_{c}^{\prime}\left(\hat{\mathbf{r}}_{k}\right) \\
& \approx \lambda^{1} \hat{m}_{a}^{\prime}+\lambda^{2}\left(\hat{\chi}_{a b}^{(d)}\right)^{\prime} B_{b}^{\prime},
\end{aligned}
$$

with

$$
\left(\hat{\chi}_{a b}^{(d)}\right)^{\prime}=\sum_{k} \frac{q_{k}^{2}}{4 m_{k}}\left(\hat{r}_{k a}^{\prime} \hat{r}_{k b}^{\prime}-\delta_{a b} \hat{r}_{k c}^{\prime} \hat{r}_{k c}^{\prime}\right)
$$

the primed diamagnetic susceptibility of the molecule.

Working to order $\lambda^{2}$ and linear order in the illuminating light, we find that the expectation values of the primed multipole moments have the following forms:

$$
\begin{aligned}
\lambda^{0}\left\langle\Psi^{\prime}\left|\hat{\mu}_{a}^{\prime}\right| \Psi^{\prime}\right\rangle & \approx \lambda^{0}\left\langle i\left|\hat{\mu}_{a}^{\prime}\right| i\right\rangle \\
& +\lambda^{0} \Re\left(\tilde{\mu}_{a}^{\prime} \mathrm{e}^{-\mathrm{i} \omega_{0} t}\right), \\
\lambda^{1}\left\langle\Psi^{\prime}\left|\hat{M}_{a}^{\prime}\right| \Psi^{\prime}\right\rangle & \approx \lambda^{1}\left\langle i\left|\hat{m}_{a}^{\prime}\right| i\right\rangle \\
& +\lambda^{1} \Re\left(\tilde{M}_{a}^{\prime} \mathrm{e}^{-\mathrm{i} \omega_{0} t}\right), \\
\lambda^{1}\left\langle\Psi^{\prime}\left|\hat{\Theta}_{a b}^{\prime}\right| \Psi^{\prime}\right\rangle & \approx \lambda^{1}\left\langle i\left|\hat{\Theta}_{a b}^{\prime}\right| i\right\rangle \\
& +\lambda^{1} \Re\left(\tilde{\Theta}_{a b}^{\prime} \mathrm{e}^{-\mathrm{i} \omega_{0} t}\right), \\
\lambda^{2}\left\langle\Psi^{\prime}\left|\hat{M}_{a b}^{\prime}\right| \Psi^{\prime}\right\rangle & \approx \lambda^{2}\left\langle i\left|\hat{m}_{a b}^{\prime}\right| i\right\rangle \\
& +\lambda^{2} \Re\left(\tilde{M}_{a b}^{\prime} \mathrm{e}^{-\mathrm{i} \omega_{0} t}\right) \\
\lambda^{2}\left\langle\Psi^{\prime}\left|\hat{Q}_{a b c}^{\prime}\right| \Psi^{\prime}\right\rangle & \approx \lambda^{2}\left\langle i\left|\hat{Q}_{a b c}^{\prime}\right| i\right\rangle \\
& +\lambda^{2} \Re\left(\tilde{Q}_{a b c}^{\prime} \mathrm{e}^{-\mathrm{i} \omega_{0} t}\right),
\end{aligned}
$$


with

$$
\begin{aligned}
\lambda^{0} \tilde{\mu}_{a}^{\prime} & \approx \lambda^{0} \alpha_{a b}^{\prime} \tilde{E}_{b}^{\prime}+\lambda^{1} \frac{1}{3} A_{a, b c}^{\prime} \partial_{c} \tilde{E}_{b}^{\prime}-\lambda^{1} \mathrm{i}\left(G_{a b}^{\prime}\right)^{\prime} \tilde{B}_{b}^{\prime} \\
& +\lambda^{2} \frac{1}{6} B_{a, b c d}^{\prime} \partial_{d} \partial_{c} \tilde{E}_{b}^{\prime}-\lambda^{2} \frac{1}{2} \mathrm{i}\left(D_{a, b c}^{(m)}\right)^{\prime} \partial_{c} \tilde{B}_{b}^{\prime},(\mathrm{B} 18) \\
\lambda^{1} \tilde{M}_{a}^{\prime} & \approx \lambda^{1} \mathrm{i}\left(G_{b a}^{\prime}\right)^{\prime} \tilde{E}_{b}^{\prime}-\lambda^{2} \frac{1}{3} \mathrm{i}\left(D_{a, b c}^{\prime}\right)^{\prime} \partial_{c} \tilde{E}_{b}^{\prime} \\
& +\lambda^{2} \chi_{a b}^{\prime} \tilde{B}_{b}^{\prime}, \\
\lambda^{1} \tilde{\Theta}_{a b}^{\prime} & \approx \lambda^{1} A_{c, a b}^{\prime} \tilde{E}_{c}^{\prime}+\lambda^{2} C_{a b, c d}^{\prime} \partial_{d} \tilde{E}_{c}^{\prime} \\
& +\lambda^{2} \mathrm{i}\left(D_{c, a b}^{\prime}\right)^{\prime} \tilde{B}_{c}^{\prime}, \\
\lambda^{2} \tilde{M}_{a b}^{\prime} & \approx \lambda^{2} \mathrm{i}\left(D_{c, a b}^{(m)^{\prime}}\right)^{\prime} \tilde{E}_{c}^{\prime} \\
\lambda^{2} \tilde{Q}_{a b c}^{\prime} & \approx \lambda^{2} B_{d, a b c}^{\prime} \tilde{E}_{d}^{\prime},
\end{aligned}
$$

where the primed reduced property tensors $\alpha_{a b}^{\prime}, A_{a, b c}^{\prime}$, $\left(G_{a b}^{\prime}\right)^{\prime}, B_{a, b c d}^{\prime},\left(D_{a, b c}^{(m)}\right)^{\prime},\left(D_{a, b c}^{\prime}\right)^{\prime}, \chi_{a b}^{\prime}$ and $C_{a b, c d}^{\prime}$ are defined identically to the unprimed reduced property tensors $\alpha_{a b}, A_{a, b c}, G_{a b}^{\prime}, B_{a, b c d}, D_{a, b c}^{(m)}, D_{a, b c}^{\prime}, \chi_{a b}$ and $C_{a b, c d}$ but with primed rather than unprimed multipole moments. Thus

$$
\begin{aligned}
\alpha_{a b}^{\prime} & =\frac{2}{\hbar} \sum_{s} \frac{\omega_{s i}}{\omega_{s i}^{2}-\omega_{0}^{2}} \mathfrak{R}\left(\left\langle i\left|\hat{\mu}_{a}^{\prime}\right| s\right\rangle\left\langle s\left|\mu_{b}^{\prime}\right| i\right\rangle\right) \\
\left(G_{a b}^{\prime}\right)^{\prime} & =-\frac{2}{\hbar} \sum_{s} \frac{\omega_{0}}{\omega_{s i}^{2}-\omega_{0}^{2}} \mathfrak{I}\left(\left\langle i\left|\hat{\mu}_{a}^{\prime}\right| s\right\rangle\left\langle s\left|\hat{m}_{b}^{\prime}\right| i\right\rangle\right),
\end{aligned}
$$

for example, where once more we have dropped explicit state exclusions from the summations [2] and refrained from using tildes for potentially complex matrix elements.
It follows that

$$
\begin{aligned}
& \alpha_{a b}^{\prime}=\alpha_{a b}, \\
& A_{a, b c}^{\prime}=A_{a, b c}-\frac{3}{2} a_{b} \alpha_{a c}-\frac{3}{2} a_{c} \alpha_{a b}+\delta_{b c} a_{d} \alpha_{a d}, \\
& \left(G_{a b}^{\prime}\right)^{\prime}=G_{a b}^{\prime}+\frac{\omega_{0}}{2} \epsilon_{b c d} a_{c} \alpha_{a d}, \\
& B_{a, b c d}^{\prime}=B_{a, b c d}+a_{b} a_{c} \alpha_{a d}+a_{b} a_{d} \alpha_{a c}+a_{c} a_{d} \alpha_{a b} \\
& -a_{b} A_{a, c d}^{(Q)}-a_{c} A_{a, b d}^{(Q)}-a_{d} A_{a, b c}^{(Q)}, \\
& C_{a b, c d}^{\prime}=C_{a b, c d}+\frac{3}{4} a_{a} a_{c} \alpha_{b d}+\frac{3}{4} a_{a} a_{d} \alpha_{b c}+\frac{3}{4} a_{b} a_{c} \alpha_{a d} \\
& +\frac{3}{4} a_{b} a_{d} \alpha_{a c}-\frac{1}{2} \delta_{a b} a_{c} a_{e} \alpha_{d e}-\frac{1}{2} \delta_{a b} a_{d} a_{e} \alpha_{c e} \\
& -\frac{1}{2} \delta_{c d} a_{a} a_{e} \alpha_{b e}-\frac{1}{2} \delta_{c d} a_{b} a_{e} \alpha_{a e}+\frac{1}{3} \delta_{a b} \delta_{c d} a_{e} a_{f} \alpha_{e f} \\
& -\frac{1}{2} a_{a} A_{b, c d}-\frac{1}{2} a_{b} A_{a, c d}-\frac{1}{2} a_{c} A_{d, a b}-\frac{1}{2} a_{d} A_{c, a b} \\
& +\frac{1}{3} \delta_{a b} a_{e} A_{e, c d}+\frac{1}{3} \delta_{c d} a_{e} A_{e, a b}, \\
& \left(D_{a, b c}^{\prime}\right)^{\prime}=D_{a, b c}^{\prime}+\frac{3 \omega_{0}}{4} \epsilon_{a d e} a_{b} a_{d} \alpha_{e c}+\frac{3 \omega_{0}}{4} \epsilon_{a d e} a_{c} a_{d} \alpha_{e b} \\
& -\frac{\omega_{0}}{2} \epsilon_{a d e} a_{d} A_{e, b c}-\frac{\omega_{0}}{2} \delta_{b c} \epsilon_{a d e} a_{d} a_{f} \alpha_{e f} \\
& +\frac{3}{2} a_{b} G_{c a}^{\prime}+\frac{3}{2} a_{c} G_{b a}^{\prime}-\delta_{b c} a_{d} G_{d a}^{\prime}, \\
& \left(D_{a, b c}^{(m)^{\prime}}\right)^{\prime}=D_{a, b c}^{(m)^{\prime}}-\frac{2 \omega_{0}}{3} \epsilon_{b d e} a_{c} a_{d} \alpha_{a e}+\frac{\omega_{0}}{3} \epsilon_{b d e} a_{d} A_{a, c e}^{(Q)} \\
& -2 a_{c} G_{a b}^{\prime}+\frac{2}{3} \delta_{b c} a_{d} G_{a d}^{(n)^{\prime}} \\
& \chi_{a b}^{\prime}=\chi_{a b}+\frac{\omega_{0}}{2} \epsilon_{a c d} a_{c} G_{d b}^{\prime}+\frac{\omega_{0}}{2} \epsilon_{b c d} a_{c} G_{d a}^{\prime} \\
& +\frac{\omega_{0}^{2}}{4} \epsilon_{a c d} \epsilon_{b e f} a_{c} a_{e} \alpha_{d f} .
\end{aligned}
$$

Note that $A_{a, b c}^{(Q)}$ and $G_{a b}^{(n)^{\prime}}$ have emerged naturally here, as anticipated in appendix A 1.

Working to order $\lambda^{2}$, we substitute (B25)-(B32) into (B18)-(B22) and Taylor expand the illuminating light about the spatial origin as

$$
\lambda^{0} E_{a}^{\prime} \approx \lambda^{0} E_{a}+\lambda^{1} a_{b} \partial_{b} E_{a}+\lambda^{2} \frac{1}{2} a_{b} a_{c} \partial_{c} \partial_{a} E_{a}
$$

for example. Comparing the results of this calculation with (7)-(11), with the latter expressed in terms of reduced property tensors using (A40)-(A52), we find that

$$
\begin{aligned}
\tilde{\mu}_{a}^{\prime} & =\tilde{\mu}_{a} \\
\tilde{\Theta}_{a b}^{\prime} & =\tilde{\Theta}_{a b}-\frac{3}{2} a_{a} \tilde{\mu}_{b}-\frac{3}{2} a_{b} \tilde{\mu}_{a}+\delta_{a b} a_{c} \tilde{\mu}_{c} \\
\tilde{m}_{a}^{\prime} & =\tilde{m}_{a}+\frac{\mathrm{i} \omega_{0}}{2} \epsilon_{a b c} a_{b} \tilde{\mu}_{c} \\
\tilde{M}_{a b}^{\prime} & =\tilde{M}_{a b}-\frac{2 \mathrm{i} \omega_{0}}{3} \epsilon_{a c d} a_{b} a_{c} \tilde{\mu}_{d}-2 a_{b} \tilde{M}_{a} \\
& +\frac{\mathrm{i} \omega_{0}}{3} \epsilon_{a c d} a_{c} \tilde{Q}_{b d}+\frac{2}{3} \delta_{a b} a_{c} \tilde{N}_{c} \\
\tilde{Q}_{a b c}^{\prime} & =\tilde{Q}_{a b c}-a_{a} \tilde{Q}_{b c}-a_{b} \tilde{Q}_{a c}-a_{c} \tilde{Q}_{a b} \\
& +a_{a} a_{b} \tilde{\mu}_{c}+a_{a} \tilde{\mu}_{b} a_{c}+\tilde{\mu}_{a} a_{b} a_{c} .
\end{aligned}
$$


Note that $\tilde{N}_{a}$ and $\tilde{Q}_{a b}$ have emerged naturally here, as anticipated in appendix A 1.

Finally, a calculation analogous to that which gives (5) and (6) [2, 34], but with a chosen as the origin of multipolar expansion, shows that the electric and magnetic fields of the scattered light have the following forms:

$$
\begin{aligned}
& \mathbf{e}^{\prime} \approx \mathfrak{R}\left(\tilde{\mathbf{e}}^{\prime} \mathrm{e}^{-\mathrm{i} \omega t}\right) \\
& \mathbf{b}^{\prime} \approx \mathfrak{R}\left(\tilde{\mathbf{b}}^{\prime} \mathrm{e}^{-\mathrm{i} \omega t}\right),
\end{aligned}
$$

with

$$
\begin{aligned}
\tilde{e}_{a}^{\prime} & \approx \frac{\mu_{0} \omega^{2} \mathrm{e}^{\mathrm{i} k(r-\hat{\mathbf{r}} \cdot \mathbf{a})}}{4 \pi r}\left(\delta_{a b}-\hat{r}_{a} \hat{r}_{b}\right)\left(\lambda^{0} \tilde{\mu}_{b}^{\prime}\right. \\
& +\lambda^{1} \frac{1}{c} \epsilon_{b c d} \tilde{M}_{c}^{\prime} \hat{r}_{d}-\lambda^{1} \frac{\mathrm{i} k}{3} \tilde{\Theta}_{b c}^{\prime} \hat{r}_{c} \\
& \left.-\lambda^{2} \frac{\mathrm{i} k}{2 c} \epsilon_{b c d} \tilde{M}_{c e}^{\prime} \hat{r}_{e} \hat{r}_{d}-\lambda^{2} \frac{k^{2}}{6} \tilde{Q}_{b c d}^{\prime} \hat{r}_{c} \hat{r}_{d}\right) \\
\tilde{b}_{a}^{\prime} & \approx \frac{1}{c} \epsilon_{a b c} \hat{r}_{b} \tilde{e}_{c}^{\prime},
\end{aligned}
$$

far from the scatterer. Working to order $\lambda^{2}$, we substitute (B34)-(B38) into (B41) and (B42) and Taylor expand the phase factor as

$$
\mathrm{e}^{\mathrm{i} k(r-\hat{\mathbf{r}} \cdot \mathbf{a})} \approx \mathrm{e}^{\mathrm{i} k r}\left[\lambda^{0}-\lambda^{1} \hat{\mathbf{r}} \cdot \mathbf{a}-\lambda^{2} \frac{1}{2} k^{2}(\hat{\mathbf{r}} \cdot \mathbf{a})^{2}\right],
$$

giving

$$
\tilde{e}_{a}^{\prime}-\tilde{e}_{a} \approx \lambda^{0} \tilde{\Delta}_{a}^{(0)}+\lambda^{1} \tilde{\Delta}_{a}^{(1)}+\lambda^{2} \tilde{\Delta}_{a}^{(2)},
$$

with $\tilde{\Delta}_{a}^{(0)}=\tilde{\Delta}_{a}^{(1)}=\tilde{\Delta}_{a}^{(2)}=0$. Thus, the scattered electric and magnetic fields are independent of our choice of origin for multipolar expansions, as they should be. It follows that

$$
\begin{aligned}
s_{\xi}^{\prime} & =\tilde{f}_{\xi a b} \tilde{e}_{a}^{\prime} \tilde{e}_{b}^{*} \\
& =s_{\xi}
\end{aligned}
$$

to order $\lambda^{2}$. Thus, the Stokes parameters of the scattered light are also independent of our choice of multipolar expansions to order $\lambda^{2}$, as they should be.

The calculations above illustrate the need to consider all of the relevant multipole moments and property tensors simultaneously: if any one of these is omitted, 'physical' predictions are obtained that depend upon our choice of origin for multipolar expansions. These predictions are meaningless, of course, as they are not unique.

\section{Appendix C: General expressions for the Stokes parameters after rotational averaging}

In this appendix we list the rotationally averaged versions of the $s_{\xi}^{(0)}$ and the $s_{\xi}^{(2)}$ for plane-wave illumination, as described in section III of the main text. We have calculated these results using the procedure described in
[22], where the rotational average $\left\langle T_{a_{1} \ldots a_{N}}\right\rangle$ of a tensor $T_{a_{1} \ldots a_{N}}$ of rank $N$ is calculated as follows:

$$
\left\langle T_{a_{1} \ldots a_{N}}\right\rangle=I_{a_{1} \ldots a_{N} a_{1}^{\prime} \ldots a_{N}^{\prime}}^{(N)} T_{a_{1}^{\prime} \ldots a_{N}^{\prime}},
$$

with $I_{a b c d a^{\prime} b^{\prime} c^{\prime} d^{\prime}}^{(4)}, I_{a b c d e a^{\prime} b^{\prime} c^{\prime} d^{\prime} e^{\prime}}^{(5)}$ and $I_{a b c d e f a^{\prime} b^{\prime} c^{\prime} d^{\prime} e^{\prime} f^{\prime}}^{(6)}$ as seen in (A2.20), (A2.24) and (A2.26) of [22]. In some of the equations below we have refrained from including tildes and superscript labels, for the sake of notational brevity. The results are quoted in terms of isotropic combinations of property tensor components, which are defined after each set of contributions. These have been defined so that they all have the same dimensions and are all real for the special case of Rayleigh scattering of far-off-resonance light by a time-reversible scatterer.

Let us emphasise that no special assumptions have been made here about the scatterer: the results below are rather general.

The ' $\alpha-\alpha$ ' terms are

$$
\left\langle s_{\xi}^{\alpha-\alpha}\right\rangle=K \Re\left(\frac{1}{40} \tilde{A}_{\xi}^{\alpha-\alpha}\right),
$$

with

$$
\begin{aligned}
\tilde{A}_{0}^{\alpha-\alpha} & =S_{0}\left(-M_{1}+14 M_{2}-M_{3}\right) \\
& +S_{0} \cos ^{2} \theta\left(3 M_{1}-2 M_{2}+3 M_{3}\right) \\
& +S_{1} \sin ^{2} \theta\left(3 M_{1}-2 M_{2}+3 M_{3}\right), \\
\tilde{A}_{1}^{\alpha-\alpha} & =S_{1}\left(-3 M_{1}+2 M_{2}-3 M_{3}\right) \\
& +S_{1} \cos ^{2} \theta\left(-3 M_{1}+2 M_{2}-3 M_{3}\right) \\
& +S_{0} \sin ^{2} \theta\left(-3 M_{1}+2 M_{2}-3 M_{3}\right), \\
\tilde{A}_{2}^{\alpha-\alpha} & =S_{2} \cos \theta\left(-6 M_{1}+4 M_{2}-6 M_{3}\right) \\
\tilde{A}_{3}^{\alpha-\alpha} & =S_{3} \cos \theta\left(10 M_{1}-10 M_{3}\right),
\end{aligned}
$$

where

$$
\begin{aligned}
\tilde{M}_{1}^{\alpha-\alpha} & =\tilde{\alpha}_{a a} \tilde{\alpha}_{b b}^{*}, \\
\tilde{M}_{2}^{\alpha-\alpha} & =\tilde{\alpha}_{a b} \tilde{\alpha}_{a b}^{*} \\
\tilde{M}_{3}^{\alpha-\alpha} & =\tilde{\alpha}_{a b} \tilde{\alpha}_{b a}^{*}
\end{aligned}
$$

are the relevant properties of the scatterer.

The ' $A-A$ ' terms are

$$
\left\langle s_{\xi}^{A-A}\right\rangle=K \Re\left[\frac{1}{7560}\left(\tilde{A}_{\xi}^{A-A}+\tilde{B}_{\xi}^{A-A}+\tilde{C}_{\xi}^{A-A}\right)\right],
$$

with

$$
\begin{aligned}
\tilde{A}_{0}^{A-A} & =S_{0}\left(3 M_{1}+3 M_{2}\right) \\
& +S_{0} \cos ^{2} \theta\left(-9 M_{1}-9 M_{2}\right) \\
& +S_{1} \sin ^{2} \theta\left(9 M_{1}+9 M_{2}\right), \\
\tilde{A}_{1}^{A-A} & =S_{1}\left(-9 M_{1}-9 M_{2}\right) \\
& +S_{1} \cos ^{2} \theta\left(-9 M_{1}-9 M_{2}\right) \\
& +S_{0} \sin ^{2} \theta\left(9 M_{1}+9 M_{2}\right), \\
\tilde{A}_{2}^{A-A} & =S_{2} \cos \theta\left(-18 M_{1}-18 M_{2}\right), \\
\tilde{A}_{3}^{A-A} & =S_{3} \cos \theta\left(14 M_{1}-14 M_{2}\right),
\end{aligned}
$$


with

$$
\begin{aligned}
\tilde{B}_{0}^{A-A}= & S_{0} \cos \theta\left(24 M_{3}-60 M_{4}\right) \\
& +S_{0} \cos ^{3} \theta\left(-40 M_{3}+16 M_{4}\right) \\
& +S_{1} \sin ^{2} \theta \cos \theta\left(-40 M_{3}+16 M_{4}\right) \\
\tilde{B}_{1}^{A-A} & =S_{1} \cos \theta\left(-32 M_{3}-4 M_{4}\right) \\
& +S_{1} \cos ^{3} \theta\left(40 M_{3}-16 M_{4}\right) \\
& +S_{0} \sin ^{2} \theta \cos \theta\left(40 M_{3}-16 M_{4}\right) \\
\tilde{B}_{2}^{A-A} & =S_{2}\left(-36 M_{3}+6 M_{4}\right) \\
& +S_{2} \cos ^{2} \theta\left(44 M_{3}-26 M_{4}\right) \\
& +\mathrm{i} S_{3} \sin ^{2} \theta\left(28 M_{3}+14 M_{4}\right) \\
\tilde{B}_{3}^{A-A}= & S_{3}\left(28 M_{3}+14 M_{4}\right) \\
& +S_{3} \cos ^{2} \theta\left(-84 M_{3}-42 M_{4}\right) \\
& +\mathrm{i} S_{2} \sin ^{2} \theta\left(-28 M_{3}-14 M_{4}\right) \\
& \\
\tilde{C}_{2}^{A-A} & =S_{2} \cos \theta\left(-18 M_{5}-18 M_{6}\right) \\
\tilde{C}_{3}^{A-A} & =S_{3} \cos \theta\left(14 M_{5}-14 M_{6}\right) \\
\tilde{C}_{0}^{A-A} & =S_{0}\left(3 M_{5}+3 M_{6}\right) \\
& +S_{0} \cos ^{2} \theta\left(-9 M_{5}-9 M_{6}\right) \\
& +S_{1} \sin ^{2} \theta\left(-9 M_{5}-9 M_{6}\right) \\
\tilde{C}_{1}^{A-A} & =S_{1}\left(-9 M_{5}-9 M_{6}\right) \\
& S_{1} \cos { }^{2} \theta\left(-9 M_{5}-9 M_{6}\right) \\
& \\
& \\
& \\
& \\
& \\
&
\end{aligned}
$$

where

$$
\begin{aligned}
& \tilde{M}_{1}^{A-A}=\frac{\omega_{0}^{2}}{c^{2}} \tilde{A}_{a, a b} \tilde{A}_{c, b c}^{*}, \\
& \tilde{M}_{2}^{A-A}=\frac{\omega_{0}^{2}}{c^{2}} \tilde{A}_{a, b c} \tilde{A}_{b, a c}^{*}, \\
& \tilde{M}_{3}^{A-A}=\frac{\omega_{0} \omega}{c^{2}} \tilde{A}_{a, a b} \tilde{\mathscr{A}}_{c, b c}^{*} \\
& \tilde{M}_{4}^{A-A}=\frac{\omega_{0} \omega}{c^{2}} \tilde{A}_{a, b c} \tilde{\mathscr{A}}_{b, a c}^{*} \\
& \tilde{M}_{5}^{A-A}=\frac{\omega^{2}}{c^{2}} \tilde{\mathscr{A}}_{a, a b} \tilde{\mathscr{A}}_{c, b c}^{*} \\
& \tilde{M}_{6}^{A-A}=\frac{\omega^{2}}{c^{2}} \tilde{\mathscr{A}}_{a, b c} \tilde{\mathscr{A}}_{b, a c}^{*}
\end{aligned}
$$

are the relevant properties of the scatterer.

The ' $G-A$ ' terms are

$$
\begin{aligned}
\left\langle s_{\xi}^{G-A}\right\rangle & =K \Re\left[\frac { 1 } { 3 6 0 } \left(\tilde{A}_{\xi}^{G-A}+\tilde{B}_{\xi}^{G-A}+\tilde{C}_{\xi}^{G-A}\right.\right. \\
& \left.\left.+\tilde{D}_{\xi}^{G-A}\right)\right]
\end{aligned}
$$

$$
\begin{aligned}
\tilde{A}_{0}^{G-A} & =S_{0}\left(2 M_{1}-4 M_{2}\right) \\
& +S_{0} \cos ^{2} \theta\left(-6 M_{1}+12 M_{2}\right) \\
& +S_{1} \sin ^{2} \theta\left(-2 M_{1}+4 M_{2}\right), \\
\tilde{A}_{1}^{G-A} & =S_{1}\left(2 M_{1}-4 M_{2}\right) \\
& +S_{1} \cos ^{2} \theta\left(2 M_{1}-4 M_{2}\right) \\
& +S_{0} \sin ^{2} \theta\left(6 M_{1}-12 M_{2}\right), \\
\tilde{A}_{2}^{G-A} & =S_{2} \cos \theta\left(4 M_{1}-8 M_{2}\right), \\
\tilde{A}_{3}^{G-A} & =S_{3} \cos \theta\left(-12 M_{1}\right),
\end{aligned}
$$

$$
\begin{aligned}
\tilde{C}_{0}^{G-A} & =S_{0} \cos \theta\left(12 M_{5}\right), \\
\tilde{C}_{1}^{G-A} & =S_{1} \cos \theta\left(-4 M_{5}+8 M_{6}\right), \\
\tilde{C}_{2}^{G-A} & =S_{2}\left(-2 M_{5}+4 M_{6}\right) \\
& +S_{2} \cos ^{2} \theta\left(-2 M_{5}+4 M_{6}\right) \\
& +\mathrm{i} S_{3} \sin ^{2} \theta\left(-6 M_{5}+12 M_{6}\right), \\
\tilde{C}_{3}^{G-A} & =S_{3}\left(-2 M_{5}+4 M_{6}\right) \\
& +S_{3} \cos ^{2} \theta\left(6 M_{5}-12 M_{6}\right) \\
& +\mathrm{i} S_{2} \sin ^{2} \theta\left(-2 M_{5}+4 M_{6}\right),
\end{aligned}
$$

$$
\begin{aligned}
\tilde{D}_{0}^{G-A} & =S_{0}\left(4 M_{7}-2 M_{8}\right) \\
& +S_{0} \cos ^{2} \theta\left(-12 M_{7}+6 M_{8}\right) \\
& +S_{1} \sin ^{2} \theta\left(-12 M_{7}+6 M_{8}\right), \\
\tilde{D}_{1}^{G-A} & =S_{1}\left(4 M_{7}-2 M_{8}\right) \\
& +S_{1} \cos ^{2} \theta\left(4 M_{7}-2 M_{8}\right) \\
& +S_{0} \sin ^{2} \theta\left(4 M_{7}-2 M_{8}\right), \\
\tilde{D}_{2}^{G-A} & =S_{2} \cos \theta\left(8 M_{7}-4 M_{8}\right) \\
\tilde{D}_{3}^{G-A} & =S_{3} \cos \theta\left(12 M_{8}\right),
\end{aligned}
$$


where

$$
\begin{aligned}
\tilde{M}_{1}^{G-A} & =\frac{\mathrm{i} \omega_{0}}{c^{2}} \epsilon_{a b c} \tilde{A}_{a, b d}^{*} \tilde{G}_{c d}, \\
\tilde{M}_{2}^{G-A} & =\frac{\mathrm{i} \omega_{0}}{c^{2}} \epsilon_{a b c} \tilde{A}_{a, b d}^{*} \tilde{G}_{d c}, \\
\tilde{M}_{3}^{G-A} & =\frac{\mathrm{i} \omega}{c^{2}} \epsilon_{a b c} \tilde{\mathscr{A}}_{a, b d}^{*} \tilde{G}_{c d}, \\
\tilde{M}_{4}^{G-A} & =\frac{\mathrm{i} \omega}{c^{2}} \epsilon_{a b c} \tilde{\mathscr{A}}_{a, b d}^{*} \tilde{G}_{d c}, \\
\tilde{M}_{5}^{G-A} & =\frac{\mathrm{i} \omega_{0}}{c^{2}} \epsilon_{a b c} \tilde{A}_{a, b d}^{*} \tilde{\mathscr{G}}_{c d}, \\
\tilde{M}_{6}^{G-A} & =\frac{\mathrm{i} \omega_{0}}{c^{2}} \epsilon_{a b c} \tilde{A}_{a, b d}^{*} \tilde{\mathscr{G}}_{d c}, \\
\tilde{M}_{7}^{G-A} & =\frac{\mathrm{i} \omega}{c^{2}} \epsilon_{a b c} \tilde{\mathscr{A}}_{a, b d}^{*} \tilde{\mathscr{G}}_{c d} \\
\tilde{M}_{8}^{G-A} & =\frac{\mathrm{i} \omega}{c^{2}} \epsilon_{a b c} \tilde{\mathscr{A}}_{a, b d}^{*} \tilde{\mathscr{G}}_{d c}
\end{aligned}
$$

are the relevant properties of the scatterer.

The ' $G-G$ ' terms are

$$
\left\langle s_{\xi}^{G-G}\right\rangle=K \Re\left[\frac{1}{120}\left(\tilde{A}_{\xi}^{G-G}+\tilde{B}_{\xi}^{G-G}+\tilde{C}_{\xi}^{G-G}\right)\right],
$$

with

$$
\begin{aligned}
& \tilde{A}_{0}^{G-G}=S_{0}\left(-M_{1}+14 M_{2}-M_{3}\right) \\
& +S_{0} \cos ^{2} \theta\left(3 M_{1}-2 M_{2}+3 M_{3}\right) \\
& +S_{1} \sin ^{2} \theta\left(-3 M_{1}+2 M_{2}-3 M_{3}\right), \\
& \tilde{A}_{1}^{G-G}=S_{1}\left(3 M_{1}-2 M_{2}+3 M_{3}\right) \\
& +S_{1} \cos ^{2} \theta\left(3 M_{1}-2 M_{2}+3 M_{3}\right) \\
& +S_{0} \sin ^{2} \theta\left(-3 M_{1}+2 M_{2}-3 M_{3}\right), \\
& \tilde{A}_{2}^{G-G}=S_{2} \cos \theta\left(6 M_{1}-4 M_{2}+6 M_{3}\right) \text {, } \\
& \tilde{A}_{3}^{G-G}=S_{3} \cos \theta\left(10 M_{1}-10 M_{3}\right), \\
& \tilde{B}_{0}^{G-G}=S_{0} \cos \theta\left(-20 M_{4}+20 M_{6}\right), \\
& \tilde{B}_{1}^{G-G}=S_{1} \cos \theta\left(-12 M_{4}+8 M_{5}-12 M_{6}\right) \text {, } \\
& \tilde{B}_{2}^{G-G}=S_{2}\left(-6 M_{4}+4 M_{5}-6 M_{6}\right) \\
& +S_{2} \cos ^{2} \theta\left(-6 M_{4}+4 M_{5}-6 M_{6}\right) \\
& +\mathrm{i} S_{3} \sin ^{2} \theta\left(-6 M_{4}+4 M_{5}-6 M_{6}\right) \text {, } \\
& \tilde{B}_{3}^{G-G}=S_{3}\left(2 M_{4}-28 M_{5}+2 M_{6}\right) \\
& +S_{3} \cos ^{2} \theta\left(-6 M_{4}+4 M_{5}-6 M_{6}\right) \\
& +\mathrm{i} S_{2} \sin ^{2} \theta\left(6 M_{4}-4 M_{5}+6 M_{6}\right) \text {, } \\
& \tilde{C}_{0}^{G-G}=S_{0}\left(-M_{7}+14 M_{8}-M_{9}\right) \\
& +S_{0} \cos ^{2} \theta\left(3 M_{7}-2 M_{8}+3 M_{9}\right) \\
& +S_{1} \sin ^{2} \theta\left(3 M_{7}-2 M_{8}+3 M_{9}\right), \\
& \tilde{C}_{1}^{G-G}=S_{1}\left(3 M_{7}-2 M_{8}+3 M_{9}\right) \\
& +S_{1} \cos ^{2} \theta\left(3 M_{7}-2 M_{8}+3 M_{9}\right) \\
& +S_{0} \sin ^{2} \theta\left(3 M_{7}-2 M_{8}+3 M_{9}\right), \\
& \tilde{C}_{2}^{G-G}=S_{2} \cos \theta\left(6 M_{7}-4 M_{8}+6 M_{9}\right) \\
& \tilde{C}_{3}^{G-G}=S_{3} \cos \theta\left(10 M_{7}-10 M_{9}\right) \text {, }
\end{aligned}
$$

where

$$
\begin{aligned}
& \tilde{M}_{1}^{G-G}=\frac{1}{c^{2}} \tilde{G}_{a a} \tilde{G}_{b b}^{*}, \\
& \tilde{M}_{2}^{G-G}=\frac{1}{c^{2}} \tilde{G}_{a b} \tilde{G}_{a b}^{*}, \\
& \tilde{M}_{3}^{G-G}=\frac{1}{c^{2}} \tilde{G}_{a b} \tilde{G}_{b a}^{*}, \\
& \tilde{M}_{4}^{G-G}=\frac{1}{c^{2}} \tilde{G}_{a a} \tilde{\mathscr{G}}_{b b}^{*}, \\
& \tilde{M}_{5}^{G-G}=\frac{1}{c^{2}} \tilde{G}_{a b} \tilde{\mathscr{G}}_{a b}^{*}, \\
& \tilde{M}_{6}^{G-G}=\frac{1}{c^{2}} \tilde{G}_{a b} \tilde{\mathscr{G}}_{b a}^{*}, \\
& \tilde{M}_{7}^{G-G}=\frac{1}{c^{2}} \tilde{\mathscr{G}}_{a a} \tilde{\mathscr{G}}_{b b}^{*}, \\
& \tilde{M}_{8}^{G-G}=\frac{1}{c^{2}} \tilde{\mathscr{G}}_{a b} \tilde{\mathscr{G}}_{a b}^{*} \\
& \tilde{M}_{9}^{G-G}=\frac{1}{c^{2}} \tilde{\mathscr{G}}_{a b} \tilde{\mathscr{G}}_{b a}^{*}
\end{aligned}
$$

are the relevant properties of the scatterer.

The ' $\alpha-B$ ' terms are

$$
\left\langle s_{\xi}^{\alpha-B}\right\rangle=K \Re\left[\frac{1}{5040}\left(\tilde{A}_{\xi}^{\alpha-B}+\tilde{B}_{\xi}^{\alpha-B}\right)\right]
$$

with

$$
\begin{aligned}
\tilde{A}_{0}^{\alpha-B} & =S_{0}\left(6 M_{1}-36 M_{2}+6 M_{3}-16 M_{4}\right) \\
& +S_{0} \cos ^{2} \theta\left(-18 M_{1}-4 M_{2}-18 M_{3}+48 M_{4}\right) \\
& +S_{1} \sin ^{2} \theta \\
& \times\left(-10 M_{1}+4 M_{2}-10 M_{3}+8 M_{4}\right) \\
\tilde{A}_{1}^{\alpha-B} & =S_{1}\left(10 M_{1}-4 M_{2}+10 M_{3}-8 M_{4}\right) \\
& +S_{1} \cos ^{2} \theta\left(10 M_{1}-4 M_{2}+10 M_{3}-8 M_{4}\right) \\
& +S_{0} \sin ^{2} \theta \\
& \times\left(18 M_{1}+4 M_{2}+18 M_{3}-48 M_{4}\right), \\
\tilde{A}_{2}^{\alpha-B} & =S_{2} \cos \theta\left(20 M_{1}-8 M_{2}+20 M_{3}-16 M_{4}\right),(\mathrm{C} 7 \\
\tilde{A}_{3}^{\alpha-B} & =S_{3} \cos \theta\left(-28 M_{1}+28 M_{3}\right),
\end{aligned}
$$

$$
\begin{aligned}
\tilde{B}_{0}^{\alpha-B} & =S_{0}\left(6 M_{5}+6 M_{6}-36 M_{7}-16 M_{8}\right) \\
& +S_{0} \cos ^{2} \theta\left(-18 M_{5}-18 M_{6}-4 M_{7}+48 M_{8}\right) \\
& +S_{1} \sin ^{2} \theta \\
& \times\left(-18 M_{5}-18 M_{6}-4 M_{7}+48 M_{8}\right), \quad(\mathrm{C} 78) \\
\tilde{B}_{1}^{\alpha-B} & =S_{1}\left(10 M_{5}+10 M_{6}-4 M_{7}-8 M_{8}\right) \\
& +S_{1} \cos ^{2} \theta\left(10 M_{5}+10 M_{6}-4 M_{7}-8 M_{8}\right) \\
& +S_{0} \sin ^{2} \theta\left(10 M_{5}+10 M_{6}-4 M_{7}-8 M_{8}\right),(\mathrm{C} 79) \\
\tilde{B}_{2}^{\alpha-B} & =S_{2} \cos \theta\left(20 M_{5}+20 M_{6}-8 M_{7}-16 M_{8}\right) \\
\tilde{B}_{3}^{\alpha-B} & =S_{3} \cos \theta\left(-28 M_{5}+28 M_{6}\right), \quad(\mathrm{C} 80)
\end{aligned}
$$


where

$$
\begin{aligned}
& \tilde{M}_{1}^{\alpha-B}=\frac{\omega_{0}}{c^{2}} \tilde{\alpha}_{a a} \tilde{B}_{b, b c c}^{*} \\
& \tilde{M}_{2}^{\alpha-B}=\frac{\omega_{0}}{c^{2}} \tilde{\alpha}_{a b} \tilde{B}_{a, b c c}^{*} \\
& \tilde{M}_{3}^{\alpha-B}=\frac{\omega_{0}}{c^{2}} \tilde{\alpha}_{a b} \tilde{B}_{b, a c c}^{*} \\
& \tilde{M}_{4}^{\alpha-B}=\frac{\omega_{0}}{c^{2}} \tilde{\alpha}_{a b} \tilde{B}_{c, a b c}^{*} \\
& \tilde{M}_{5}^{\alpha-B}=\frac{\omega}{c^{2}} \tilde{\alpha}_{a a} \tilde{\mathscr{B}}_{b, b c c}^{*} \\
& \tilde{M}_{6}^{\alpha-B}=\frac{\omega}{c^{2}} \tilde{\alpha}_{a b} \tilde{\mathscr{B}}_{a, b c c}^{*} \\
& \tilde{M}_{7}^{\alpha-B}=\frac{\omega}{c^{2}} \tilde{\alpha}_{a b} \tilde{\mathscr{B}}_{b, a c c}^{*} \\
& \tilde{M}_{8}^{\alpha-B}=\frac{\omega}{c^{2}} \tilde{\alpha}_{a b} \tilde{\mathscr{B}}_{c, a b c}^{*}
\end{aligned}
$$

are the relevant properties of the scatterer.

The ' $\alpha-C$ ' terms are

$$
\left\langle s_{\xi}^{\alpha-C}\right\rangle=K \Re\left(\frac{1}{1260} \tilde{A}_{\xi}^{\alpha-C}\right)
$$

with

$$
\begin{aligned}
\tilde{A}_{0}^{\alpha-C} & =S_{0} \cos \theta\left(-6 M_{1}+30 M_{2}-12 M_{3}\right) \\
& +S_{0} \cos ^{3} \theta\left(10 M_{1}-8 M_{2}+20 M_{3}\right) \\
& +S_{1} \sin ^{2} \theta \cos \theta\left(10 M_{1}-8 M_{2}+20 M_{3}\right), \\
\tilde{A}_{1}^{\alpha-C} & =S_{1} \cos \theta\left(-6 M_{1}+2 M_{2}+16 M_{3}\right) \\
& +S_{1} \cos ^{3} \theta\left(-10 M_{1}+8 M_{2}-20 M_{3}\right) \\
& +S_{0} \sin ^{2} \theta \cos \theta\left(-10 M_{1}+8 M_{2}-20 M_{3}\right) \\
\tilde{A}_{2}^{\alpha-C} & =S_{2}\left(2 M_{1}-3 M_{2}+18 M_{3}\right) \\
& +S_{2} \cos ^{2} \theta\left(-18 M_{1}+13 M_{2}-22 M_{3}\right) \\
& +\mathrm{i} S_{3} \sin ^{2} \theta\left(-14 M_{1}+7 M_{2}+14 M_{3}\right) \\
\tilde{A}_{3}^{\alpha-C} & =S_{3}\left(-14 M_{1}+7 M_{2}+14 M_{3}\right) \\
& +S_{3} \cos ^{2} \theta\left(42 M_{1}-21 M_{2}-42 M_{3}\right) \\
& +\mathrm{i} S_{2} \sin ^{2} \theta\left(-14 M_{1}+7 M_{2}+14 M_{3}\right),
\end{aligned}
$$

where

$$
\begin{aligned}
& \tilde{M}_{1}^{\alpha-C}=\frac{\omega \omega_{0}}{c^{2}} \tilde{\alpha}_{a a} \tilde{C}_{b c, b c}^{*}, \\
& \tilde{M}_{2}^{\alpha-C}=\frac{\omega \omega_{0}}{c^{2}} \tilde{\alpha}_{a b} \tilde{C}_{a c, b c}^{*} \\
& \tilde{M}_{3}^{\alpha-C}=\frac{\omega \omega_{0}}{c^{2}} \tilde{\alpha}_{a b} \tilde{C}_{b c, a c}^{*}
\end{aligned}
$$

are the relevant properties of the scatterer.

The ' $\alpha-D$ ' terms are

$$
\left\langle s_{\xi}^{\alpha-D}\right\rangle=K \Re\left(\frac{1}{180} \tilde{A}_{\xi}^{\alpha-D}\right),
$$

with

$$
\begin{aligned}
\tilde{A}_{0}^{\alpha-D} & =S_{0} \cos \theta\left(-6 M_{1}+6 M_{2}-6 M_{3}\right), \\
\tilde{A}_{1}^{\alpha-D} & =S_{1} \cos \theta\left(-2 M_{1}-2 M_{2}+2 M_{3}-4 M_{4}\right), \\
\tilde{A}_{2}^{\alpha-D} & =S_{2}\left(-M_{1}-M_{2}+M_{3}-2 M_{4}\right) \\
& +S_{2} \cos ^{2} \theta\left(-M_{1}-M_{2}+M_{3}-2 M_{4}\right) \\
& +\mathrm{i} S_{3} \sin ^{2} \theta\left(M_{1}+M_{2}+3 M_{3}-6 M_{4}\right) \\
\tilde{A}_{3}^{\alpha-D} & =S_{3}\left(M_{1}+M_{2}-M_{3}+2 M_{4}\right) \\
& +S_{3} \cos ^{2} \theta\left(-3 M_{1}-3 M_{2}+3 M_{3}-6 M_{4}\right) \\
& +\mathrm{i} S_{2} \sin ^{2} \theta\left(-3 M_{1}-3 M_{2}-M_{3}+2 M_{4}\right),
\end{aligned}
$$

where

$$
\begin{aligned}
& \tilde{M}_{1}^{\alpha-D}=\frac{\mathrm{i} \omega}{c^{2}} \epsilon_{a b c} \tilde{\alpha}_{a b} \tilde{\mathscr{D}}_{d, c d}^{*}, \\
& \tilde{M}_{2}^{\alpha-D}=\frac{\mathrm{i} \omega}{c^{2}} \epsilon_{a b c} \tilde{\alpha}_{a d} \tilde{\mathscr{D}}_{b, c d}^{*}, \\
& \tilde{M}_{3}^{\alpha-D}=\frac{\mathrm{i} \omega_{0}}{c^{2}} \epsilon_{a b c} \tilde{\alpha}_{a d} \tilde{D}_{b, c d}^{*} \\
& \tilde{M}_{4}^{\alpha-D}=\frac{\mathrm{i} \omega_{0}}{c^{2}} \epsilon_{a b c} \tilde{\alpha}_{a b} \tilde{D}_{d, c d}^{*}
\end{aligned}
$$

are the relevant properties of the scatterer.

The ' $\alpha-D^{m}$ ' terms are

$$
\left\langle s_{\xi}^{\alpha-D^{m}}\right\rangle=K \Re\left[\frac{1}{120}\left(\tilde{A}_{\xi}^{\alpha-D^{m}}+\tilde{B}_{\xi}^{\alpha-D^{m}}\right)\right],
$$

with

$$
\begin{aligned}
\tilde{A}_{0}^{\alpha-D^{m}} & =S_{0}\left(M_{1}-8 M_{2}+6 M_{3}+M_{4}-6 M_{6}\right) \\
& +S_{0} \cos ^{2} \theta\left(-3 M_{1}+4 M_{2}+2 M_{3}-3 M_{4}-2 M_{6}\right) \\
& +S_{1} \sin ^{2} \theta \\
& \times\left(M_{1}-2 M_{3}-M_{4}+2 M_{5}-2 M_{6}\right), \quad(\mathrm{C} 99) \\
\tilde{A}_{1}^{\alpha-D^{m}} & =S_{1}\left(-M_{1}+2 M_{3}+M_{4}-2 M_{5}+2 M_{6}\right) \\
& +S_{1} \cos ^{2} \theta\left(-M_{1}+2 M_{3}+M_{4}-2 M_{5}+2 M_{6}\right) \\
& +S_{0} \sin ^{2} \theta \\
& \times\left(3 M_{1}-4 M_{2}-2 M_{3}+3 M_{4}+2 M_{6}\right), \quad(\mathrm{C} 100) \\
\tilde{A}_{2}^{\alpha-D^{m}} & =S_{2} \cos \theta \\
& \times\left(-2 M_{1}+4 M_{3}+2 M_{4}-4 M_{5}+4 M_{6}\right), \quad(\mathrm{C} 101) \\
\tilde{A}_{3}^{\alpha-D^{m}} & =S_{3} \cos \theta\left(2 M_{1}-2 M_{4}+8 M_{5}\right), \quad(\mathrm{C} 102)
\end{aligned}
$$

$$
\begin{aligned}
\tilde{B}_{0}^{\alpha-D^{m}} & =S_{0}\left(-M_{8}-M_{10}+6 M_{12}\right) \\
& +S_{0} \cos ^{2} \theta\left(3 M_{8}+3 M_{10}+2 M_{12}\right) \\
& +S_{1} \sin ^{2} \theta\left(3 M_{8}+3 M_{10}+2 M_{12}\right), \quad(\mathrm{C} 103) \\
\tilde{B}_{1}^{\alpha-D^{m}} & =S_{1}\left(-M_{8}+2 M_{9}-M_{10}+2 M_{11}-2 M_{12}\right) \\
& +S_{1} \cos ^{2} \theta\left(-M_{8}+2 M_{9}-M_{10}+2 M_{11}-2 M_{12}\right) \\
& +S_{0} \sin ^{2} \theta \\
& \times\left(-M_{8}+2 M_{9}-M_{10}+2 M_{11}-2 M_{12}\right), \quad(\mathrm{C} 104) \\
& =S_{2} \cos \theta \\
\tilde{B}_{2}^{\alpha-D^{m}} & \times\left(-2 M_{8}+4 M_{9}-2 M_{10}+4 M_{11}-4 M_{12}\right) \quad(\mathrm{C} 105) \\
\tilde{B}_{3}^{\alpha-D^{m}} & =S_{3} \cos \theta \\
& \times\left(-4 M_{7}-2 M_{8}+8 M_{9}+2 M_{10}-8 M_{11}\right), \quad(\mathrm{C} 106)
\end{aligned}
$$


where

$$
\begin{aligned}
& \tilde{M}_{1}^{\alpha-D^{m}}=\frac{\mathrm{i} \omega_{0}}{c^{2}} \epsilon_{a b c} \tilde{\alpha}_{a b} \tilde{D}_{c, d d}^{(m) *}, \\
& \tilde{M}_{2}^{\alpha-D^{m}}=\frac{\mathrm{i} \omega_{0}}{c^{2}} \epsilon_{a b c} \tilde{\alpha}_{a b} \tilde{D}_{d, c d}^{(m) *}, \\
& \tilde{M}_{3}^{\alpha-D^{m}}=\frac{\mathrm{i} \omega_{0}}{c^{2}} \epsilon_{a b c} \tilde{\alpha}_{a b} \tilde{D}_{d, d c}^{(m) *}, \\
& \tilde{M}_{4}^{\alpha-D^{m}}=\frac{\mathrm{i} \omega_{0}}{c^{2}} \epsilon_{a b c} \tilde{\alpha}_{a d} \tilde{D}_{b, c d}^{(m) *}, \\
& \tilde{M}_{5}^{\alpha-D^{m}}=\frac{\mathrm{i} \omega_{0}}{c^{2}} \epsilon_{a b c} \tilde{\alpha}_{a d} \tilde{D}_{b, d c}^{(m) *}, \\
& \tilde{M}_{6}^{\alpha-D^{m}}=\frac{\mathrm{i} \omega_{0}}{c^{2}} \epsilon_{a b c} \tilde{\alpha}_{a d} \tilde{D}_{d, b c}^{(m) *}, \\
& \tilde{M}_{7}^{\alpha-D^{m}}=\frac{\mathrm{i} \omega}{c^{2}} \epsilon_{a b c} \tilde{\alpha}_{a b} \tilde{\mathscr{D}}_{c, d d}^{(m) *}, \\
& \tilde{M}_{8}^{\alpha-D^{m}}=\frac{\mathrm{i} \omega}{c^{2}} \epsilon_{a b c} \tilde{\alpha}_{a b} \tilde{\mathscr{D}}_{d, c d}^{(m) *}, \\
& \tilde{M}_{9}^{\alpha-D^{m}}=\frac{\mathrm{i} \omega}{c^{2}} \epsilon_{a b c} \tilde{\alpha}_{a b} \tilde{\mathscr{D}}_{d, d c}^{(m) *}, \\
& \tilde{M}_{10}^{\alpha-D^{m}}=\frac{\mathrm{i} \omega}{c^{2}} \epsilon_{a b c} \tilde{\alpha}_{a d} \tilde{\mathscr{D}}_{b, c d}^{(m) *}, \\
& \tilde{M}_{11}^{\alpha-D^{m}}=\frac{\mathrm{i} \omega}{c^{2}} \epsilon_{a b c} \tilde{\alpha}_{a d} \tilde{\mathscr{D}}_{b, d c}^{(m) *} \\
& \tilde{M}_{12}^{\alpha-D^{m}}=\frac{\mathrm{i} \omega}{c^{2}} \epsilon_{a b c} \tilde{\alpha}_{a d} \tilde{\mathscr{D}}_{d, b c}^{(m) *}
\end{aligned}
$$

are the relevant properties of the scatterer.

The general ' $\alpha-\chi$ ' terms are

$$
\left\langle s_{\xi}^{\alpha-\chi}\right\rangle=K \Re\left(\frac{1}{60} \tilde{A}_{\xi}^{\alpha-\chi}\right),
$$

with

$$
\begin{aligned}
\tilde{A}_{0}^{\alpha-\chi} & =S_{0} \cos \theta\left(10 M_{1}-10 M_{3}\right), \\
\tilde{A}_{1}^{\alpha-\chi} & =S_{1} \cos \theta\left(-6 M_{1}+4 M_{2}-6 M_{3}\right), \\
\tilde{A}_{2}^{\alpha-\chi} & =S_{2}\left(-3 M_{1}+2 M_{2}-3 M_{3}\right) \\
& +S_{2} \cos ^{2} \theta\left(-3 M_{1}+2 M_{2}-3 M_{3}\right) \\
& +\mathrm{i} S_{3} \sin ^{2} \theta\left(3 M_{1}-2 M_{2}+3 M_{3}\right) \\
\tilde{A}_{3}^{\alpha-\chi} & =S_{3}\left(-M_{1}+14 M_{2}-M_{3}\right) \\
& +S_{3} \cos ^{2} \theta\left(3 M_{1}-2 M_{2}+3 M_{3}\right) \\
& +\mathrm{i} S_{2} \sin ^{2} \theta\left(3 M_{1}-2 M_{2}+3 M_{3}\right),
\end{aligned}
$$

where

$$
\begin{aligned}
& \tilde{M}_{1}^{\alpha-\chi}=\frac{1}{c^{2}} \tilde{\alpha}_{a a} \tilde{\chi}_{b b}^{*} \\
& \tilde{M}_{2}^{\alpha-\chi}=\frac{1}{c^{2}} \tilde{\alpha}_{a b} \tilde{\chi}_{a b}^{*} \\
& \tilde{M}_{3}^{\alpha-\chi}=\frac{1}{c^{2}} \tilde{\alpha}_{a b} \tilde{\chi}_{b a}^{*}
\end{aligned}
$$

are the relevant properties of the scatterer.
Appendix D: Explicit expressions for the coefficients $\mathrm{A}, \ldots \mathrm{R}^{\prime}$

In this appendix we list explicit expressions for the coefficients $\mathrm{A}, \ldots, \mathrm{R}^{\prime}$.

The unprimed coefficients are

$$
\begin{aligned}
\mathrm{A} & =\frac{1}{40}\left(-\alpha_{a a} \alpha_{b b}+13 \alpha_{a b} \alpha_{a b}\right) \\
& +\frac{k_{0}^{2}}{7560}\left(6 A_{a, a b} A_{c, b c}+6 A_{a, b c} A_{b, a c}\right) \\
& +\frac{k_{0}}{360 c}\left(4 \epsilon_{a b c} A_{a, b d} G_{c d}^{\prime}-8 \epsilon_{a b c} A_{a, b d} G_{d c}^{\prime}\right) \\
& +\frac{1}{120 c^{2}}\left(-2 G_{a a}^{\prime} G_{b b}^{\prime}+24 G_{a b}^{\prime} G_{a b}^{\prime}-2 G_{a b}^{\prime} G_{b a}^{\prime}\right) \\
& +\frac{k_{0}}{5040 c}\left(12 \alpha_{a a} B_{b, b c c}-60 \alpha_{a b} B_{a, b c c}-32 \alpha_{a b} B_{c, a b c}\right) \\
& +\frac{k_{0}}{120 c}\left(2 \epsilon_{a b c} \alpha_{a d} D_{b, c d}^{(m)^{\prime}}-12 \epsilon_{a b c} \alpha_{a d} D_{d, b c}^{(m)^{\prime}}\right),
\end{aligned}
$$

$$
\begin{aligned}
\mathrm{C} & =\frac{1}{40}\left(3 \alpha_{a a} \alpha_{b b}+\alpha_{a b} \alpha_{a b}\right) \\
& +\frac{k_{0}^{2}}{7560}\left(-18 A_{a, a b} A_{c, b c}-18 A_{a, b c} A_{b, a c}\right) \\
& +\frac{k_{0}}{360 c}\left(-12 \epsilon_{a b c} A_{a, b d} G_{c d}^{\prime}+24 \epsilon_{a b c} A_{a, b d} G_{d c}^{\prime}\right) \\
& +\frac{1}{120 c^{2}}\left(6 G_{a a}^{\prime} G_{b b}^{\prime}-4 G_{a b}^{\prime} G_{a b}^{\prime}+6 G_{a b}^{\prime} G_{b a}^{\prime}\right) \\
& +\frac{k_{0}}{5040 c}\left(-36 \alpha_{a a} B_{b, b c c}-44 \alpha_{a b} B_{a, b c c}+96 \alpha_{a b} B_{c, a b c}\right) \\
& +\frac{k_{0}}{120 c}\left(-\epsilon_{a b c} \alpha_{a d} D_{b, c d}^{(m)^{\prime}}+8 \epsilon_{a b c} \alpha_{a d} D_{d, b c}^{(m)^{\prime}}\right)
\end{aligned}
$$

$$
\begin{aligned}
\mathrm{E} & =-\mathrm{K}=\frac{1}{40}\left(3 \alpha_{a a} \alpha_{b b}+\alpha_{a b} \alpha_{a b}\right) \\
& +\frac{k_{0}}{360 c}\left(-8 \epsilon_{a b c} A_{a, b d} G_{c d}^{\prime}+16 \epsilon_{a b c} A_{a, b d} G_{d c}^{\prime}\right) \\
& +\frac{k_{0}}{5040 c}\left(-28 \alpha_{a a} B_{b, b c c}-28 \alpha_{a b} B_{a, b c c}+56 \alpha_{a b} B_{c, a b c}\right) \\
& +\frac{k_{0}}{120 c}\left(4 \epsilon_{a b c} \alpha_{a d} D_{b, c d}^{(m)^{\prime}}+4 \epsilon_{a b c} \alpha_{a d} D_{d, b c}^{(m)^{\prime}}\right. \\
& \left.-2 \epsilon_{a b c} \alpha_{a d} D_{b, d c}^{(m)^{\prime}}\right)
\end{aligned}
$$




$$
\begin{aligned}
& \mathrm{G}=\mathrm{I}=\frac{1}{40}\left(-3 \alpha_{a a} \alpha_{b b}-\alpha_{a b} \alpha_{a b}\right) \\
& +\frac{k_{0}^{2}}{7560}\left(-18 A_{a, a b} A_{c, b c}-18 A_{a, b c} A_{b, a c}\right) \\
& +\frac{k_{0}}{360 c}\left(4 \epsilon_{a b c} A_{a, b d} G_{c d}^{\prime}-8 \epsilon_{a b c} A_{a, b d} G_{d c}^{\prime}\right) \\
& +\frac{1}{120 c^{2}}\left(6 G_{a a}^{\prime} G_{b b}^{\prime}-4 G_{a b}^{\prime} G_{a b}^{\prime}+6 G_{a b}^{\prime} G_{b a}^{\prime}\right) \\
& +\frac{k_{0}}{5040 c}\left(20 \alpha_{a a} B_{b, b c c}+12 \alpha_{a b} B_{a, b c c}-16 \alpha_{a b} B_{c, a b c}\right) \\
& +\frac{k_{0}}{120 c}\left(-2 \epsilon_{a b c} \alpha_{a d} D_{b, c d}^{(m)^{\prime}}-4 \epsilon_{a b c} \alpha_{a d} D_{d, b c}^{(m)^{\prime}}\right. \\
& \left.+4 \epsilon_{a b c} \alpha_{a d} D_{b, d c}^{(m)^{\prime}}\right) \\
& \mathrm{N}=\frac{1}{40}\left(-6 \alpha_{a a} \alpha_{b b}-2 \alpha_{a b} \alpha_{a b}\right) \\
& +\frac{k_{0}^{2}}{7560}\left(-36 A_{a, a b} A_{c, b c}-36 A_{a, b c} A_{b, a c}\right) \\
& +\frac{k_{0}}{360 c}\left(8 \epsilon_{a b c} A_{a, b d} G_{c d}^{\prime}-16 \epsilon_{a b c} A_{a, b d} G_{d c}^{\prime}\right) \\
& +\frac{1}{120 c^{2}}\left(12 G_{a a}^{\prime} G_{b b}^{\prime}-8 G_{a b}^{\prime} G_{a b}^{\prime}+12 G_{a b}^{\prime} G_{b a}^{\prime}\right) \\
& +\frac{k_{0}}{5040 c}\left(40 \alpha_{a a} B_{b, b c c}+24 \alpha_{a b} B_{a, b c c}-32 \alpha_{a b} B_{c, a b c}\right) \\
& +\frac{k_{0}}{120 c}\left(-4 \epsilon_{a b c} \alpha_{a d} D_{b, c d}^{(m)^{\prime}}-8 \epsilon_{a b c} \alpha_{a d} D_{d, b c}^{(m)^{\prime}}\right. \\
& \left.+8 \epsilon_{a b c} \alpha_{a d} D_{b, d c}^{(m)^{\prime}}\right) \\
& \mathrm{Q}=\frac{1}{40}\left(10 \alpha_{a a} \alpha_{b b}-10 \alpha_{a b} \alpha_{a b}\right) \\
& +\frac{k_{0}^{2}}{7560}\left(28 A_{a, a b} A_{c, b c}-28 A_{a, b c} A_{b, a c}\right) \\
& +\frac{k_{0}}{360 c}\left(-24 \epsilon_{a b c} A_{a, b d} G_{c d}^{\prime}\right) \\
& +\frac{1}{120 c^{2}}\left(20 G_{a a}^{\prime} G_{b b}^{\prime}-20 G_{a b}^{\prime} G_{b a}^{\prime}\right) \\
& +\frac{k_{0}}{5040 c}\left(-56 \alpha_{a a} B_{b, b c c}+56 \alpha_{a b} B_{a, b c c}-32 \alpha_{a b} B_{c, a b c}\right) \\
& +\frac{k_{0}}{120 c}\left(4 \epsilon_{a b c} \alpha_{a d} D_{b, c d}^{(m)^{\prime}}-16 \epsilon_{a b c} \alpha_{a d} D_{b, d c}^{(m)^{\prime}}\right) .
\end{aligned}
$$

The singly-primed coefficients are

$$
\begin{aligned}
\mathrm{B}^{\prime} & =\frac{k_{0}^{2}}{7560}\left(24 A_{a, a b} A_{c, b c}-60 A_{a, b c} A_{b, a c}\right) \\
& +\frac{k_{0}}{360 c}\left(-24 \epsilon_{a b c} A_{a, b d} G_{d c}^{\prime}\right) \\
& +\frac{1}{120 c^{2}}\left(20 G_{a a}^{\prime} G_{b b}^{\prime}-20 G_{a b}^{\prime} G_{a b}^{\prime}\right) \\
& +\frac{k_{0}^{2}}{1260}\left(-6 \alpha_{a a} C_{b c, b c}+28 \alpha_{a b} C_{a c, b c}\right) \\
& +\frac{\omega_{0}}{180 c^{2}}\left(-12 \epsilon_{a b c} \alpha_{a d} D_{b, c d}^{\prime}\right) \\
& +\frac{1}{60 c^{2}}\left(10 \alpha_{a a} \chi_{b b}-10 \alpha_{a b} \chi_{a b}\right)
\end{aligned}
$$

$$
\begin{aligned}
\mathrm{H}^{\prime} & =\frac{k_{0}^{2}}{7560}\left(-3 A_{a, a b} A_{c, b c}-4 A_{a, b c} A_{b, a c}\right) \\
& +\frac{k_{0}}{360 c}\left(-16 \epsilon_{a b c} A_{a, b d} G_{c d}^{\prime}+8 \epsilon_{a b c} A_{a, b d} G_{d c}^{\prime}\right) \\
& +\frac{1}{120 c^{2}}\left(12 G_{a a}^{\prime} G_{b b}^{\prime}+12 G_{a b}^{\prime} G_{a b}^{\prime}-8 G_{a b}^{\prime} G_{b a}^{\prime}\right) \\
& +\frac{k_{0}^{2}}{1260}\left(-6 \alpha_{a a} C_{b c, b c}+18 \alpha_{a b} C_{a c, b c}\right) \\
& +\frac{k_{0}}{180 c}\left(-4 \epsilon_{a b c} \alpha_{a d} D_{b, c d}^{\prime}\right) \\
& +\frac{1}{60 c^{2}}\left(-6 \alpha_{a a} \chi_{b b}-2 \alpha_{a b} \chi_{a b}\right)
\end{aligned}
$$

$$
\begin{aligned}
\mathrm{M}^{\prime} & =\frac{k_{0}^{2}}{7560}\left(-36 A_{a, a b} A_{c, b c}+6 A_{a, b c} A_{b, a c}\right) \\
& +\frac{k_{0}}{360 c}\left(-8 \epsilon_{a b c} A_{a, b d} G_{c d}^{\prime}+4 \epsilon_{a b c} A_{a, b d} G_{d c}^{\prime}\right) \\
& +\frac{1}{120 c^{2}}\left(6 G_{a a}^{\prime} G_{b b}^{\prime}+6 G_{a b}^{\prime} G_{a b}^{\prime}-4 G_{a b}^{\prime} G_{b a}^{\prime}\right) \\
& +\frac{k_{0}^{2}}{1260}\left(2 \alpha_{a a} C_{b c, b c}+15 \alpha_{a b} C_{a c, b c}\right) \\
& +\frac{k_{0}}{180 c}\left(-2 \epsilon_{a b c} \alpha_{a d} D_{b, c d}^{\prime}\right) \\
& +\frac{1}{60 c^{2}}\left(-3 \alpha_{a a} \chi_{b b}-\alpha_{a b} \chi_{a b}\right),
\end{aligned}
$$

$$
\begin{aligned}
0^{\prime} & =\frac{k_{0}^{2}}{7560}\left(44 A_{a, a b} A_{c, b c}-26 A_{a, b c} A_{b, a c}\right) \\
& +\frac{k_{0}}{360 c}\left(-8 \epsilon_{a b c} A_{a, b d} G_{c d}^{\prime}+4 \epsilon_{a b c} A_{a, b d} G_{d c}^{\prime}\right) \\
& +\frac{1}{120 c^{2}}\left(6 G_{a a}^{\prime} G_{b b}^{\prime}+6 G_{a b}^{\prime} G_{a b}^{\prime}-4 G_{a b}^{\prime} G_{b a}^{\prime}\right) \\
& +\frac{k_{0}^{2}}{1260}\left(-18 \alpha_{a a} C_{b c, b c}-9 \alpha_{a b} C_{a c, b c}\right) \\
& +\frac{k_{0}}{180 c}\left(-2 \epsilon_{a b c} \alpha_{a d} D_{b, c d}^{\prime}\right) \\
& +\frac{1}{60 c^{2}}\left(-3 \alpha_{a a} \chi_{b b}-\alpha_{a b} \chi_{a b}\right)
\end{aligned}
$$

$$
\begin{aligned}
\mathrm{P}^{\prime} & =\frac{k_{0}^{2}}{7560}\left(28 A_{a, a b} A_{c, b c}+14 A_{a, b c} A_{b, a c}\right) \\
& +\frac{k_{0}}{360 c}\left(-8 \epsilon_{a b c} A_{a, b d} G_{c d}^{\prime}+4 \epsilon_{a b c} A_{a, b d} G_{d c}^{\prime}\right) \\
& +\frac{1}{120 c^{2}}\left(-2 G_{a a}^{\prime} G_{b b}^{\prime}-2 G_{a b}^{\prime} G_{a b}^{\prime}+28 G_{a b}^{\prime} G_{b a}^{\prime}\right) \\
& +\frac{k_{0}^{2}}{1260}\left(-14 \alpha_{a a} C_{b c, b c}+21 \alpha_{a b} C_{a c, b c}\right) \\
& +\frac{k_{0}}{180 c}\left(2 \epsilon_{a b c} \alpha_{a d} D_{b, c d}^{\prime}\right) \\
& +\frac{1}{60 c^{2}}\left(-\alpha_{a a} \chi_{b b}+13 \alpha_{a b} \chi_{a b}\right)
\end{aligned}
$$




$$
\begin{aligned}
\mathrm{R}^{\prime} & =\frac{k_{0}^{2}}{7560}\left(-84 A_{a, a b} A_{c, b c}-42 A_{a, b c} A_{b, a c}\right) \\
& +\frac{k_{0}}{360 c}\left(24 \epsilon_{a b c} A_{a, b d} G_{c d}^{\prime}-12 \epsilon_{a b c} A_{a, b d} G_{d c}^{\prime}\right) \\
& +\frac{1}{120 c^{2}}\left(6 G_{a a}^{\prime} G_{b b}^{\prime}+6 G_{a b}^{\prime} G_{a b}^{\prime}-4 G_{a b}^{\prime} G_{b a}^{\prime}\right) \\
& +\frac{k_{0}^{2}}{1260}\left(42 \alpha_{a a} C_{b c, b c}-63 \alpha_{a b} C_{a c, b c}\right) \\
& +\frac{k_{0}}{180 c}\left(-6 \epsilon_{a b c} \alpha_{a d} D_{b, c d}^{\prime}\right) \\
& +\frac{1}{60 c^{2}}\left(3 \alpha_{a a} \chi_{b b}+\alpha_{a b} \chi_{a b}\right) .
\end{aligned}
$$

Finally, the doubly-primed coefficients are

$$
\begin{aligned}
\mathrm{D}^{\prime \prime} & =\mathrm{F}^{\prime \prime}=-\mathrm{J}^{\prime \prime}=-\mathrm{L}^{\prime \prime} \\
& =\frac{k_{0}^{2}}{7560}\left(-40 A_{a, a b} A_{c, b c}+16 A_{a, b c} A_{b, a c}\right) \\
& +\frac{k_{0}^{2}}{1260}\left(10 \alpha_{a a} C_{b c, b c}+12 \alpha_{a b} C_{a c, b c}\right) .
\end{aligned}
$$

[1] H. C. van de Hulst. Light Scattering by Small Particles. Dover Publications, 1981.

[2] L. D. Barron. Molecular Light Scattering and Optical Activity. Cambridge University Press, 2004.

[3] E. M. Purcell and C. R. Pennypacker. Scattering and absorption of light by nonspherical dielectric grains. Astrophys. J., 186:705-714, 1973.

[4] D. C. Aronstein and A. C. Piccirillo. Have Blue and the F-117A: Evolution of the "Stealth Fighter". American Institute of Aeronautics \& Astronautics, 1997.

[5] E. W. Blanch, L. Hecht, C. D. Syme, V. Volpetti, G. P. Lomonossoff, K. Nielsen, and L. D. Barron. Molecular structures of viruses from Raman optical activity. Gen. Virol., 83:2593-2600, 2002.

[6] X. Zhang, L. Hu and M. X. He. Scattering by pure seawater: effect of salinity. Opt. Exp., 17:5698-5710, 2009.

[7] F. Papoff and B. Hourahine. Geometrical Mie theory for resonances in nanoparticles of any shape. Opt. Exp., 19:2143-21444, 2011.

[8] K. Hiramatsu, M. Okuno, H. Kano, P. Leproux, V. Couderc and H. Hamaguchi. Observation of Raman optical activity by heterodyne-detected polarization-resolved coherent anti-Stokes Raman scattering. Phys. Rev. Lett., 109:083901, 2012.

[9] M. D. Williams, J. S. Ford and D. L. Andrews. HyperRayleigh scattering in centrosymmetric systems. $J$. Chem. Phys., 143:124301, 2015.

[10] L. Wang and X. G. Xu. Scattering-type scanning nearfield optical microscopy with reconstruction of vertical interaction. Nat. Comm., 6:8973, 2015.

[11] K. A. Forbes, D. S. Bradshaw and D. L. Andrews. Identifying diamagnetic interactions in scattering and nonlinear optics. Phys. Rev. A, 94:033837, 2016.

[12] M. Nieto-Vesperinas. Chiral optical fields: a unified formulation of helicity scattered from particles and dichroism enhancement. Phil. Trans. R. Soc. A, 375:20160314, 2017.
[13] D. McArthur, B. Hourahine and F. Papoff. Enhancing ultraviolet spontaneous emission with a designed quantum vacuum. Opt. Exp., 25:4162, 2017.

[14] A. Saraiva, F. S. de Aguiar Júnior, R. de Melo e Souza, A. P. Pena, C. H. Monken, M. F. Santos, B. Koiller and A. Jorio. Photonic counterparts of Cooper pairs. Phys. Rev. Lett., 119:193603, 2017.

[15] S. Ahrens and C. P. Sun. Spin in Compton scattering with pronounced polarization dynamics. Phys. Rev. A, 96:063407, 2017.

[16] Hon. J. W. Strutt. On the light from the sky, its polarization and colour. Phil. Mag., 41:107-120, 1871.

[17] L. Brillouin. Diffusion de la lumière et des rayons X par un corps transparent homogène - Influence de l'agitation thermique. Annales de Physique, 9:88-122, 1922.

[18] A. H. Compton. A quantum theory of the scattering of x-rays by light elements. Phys. Rev., 21:483-502, 1923.

[19] A. Smekal. Zur Quantentheorie der Dispersion. Naturwissenschaften, 11:873-875, 1923.

[20] G. S. Landsberg and L. Mandelstam. Über die Lichtzerstreuung in Kristallen. Zeitschrift für Physik, 50:769-780, 1928.

[21] C. V. Raman. A new radiation. Indian J. Phys., 2:387398, 1928.

[22] D. P. Craig and T. Thirunamachandran. Molecular Quantum Electrodynamics: An Introduction to Radiation Molecule Interactions. Dover Publications, 1998.

[23] Hon. J. W. Strutt. On the light from the sky, its polarization and colour. Phil. Mag., 41:274-279, 1871.

[24] Hon. J. W. Strutt. On the light from the sky, its polarization and colour. Phil. Mag., 41:447-454, 1871.

[25] G. Mie. Beiträge zur Optik trüber Medien, speziell kolloidaler Metallösungen. Annalen der Physik, 330:377335, 1908.

[26] P. W. Atkins and L. D. Barron. Rayleigh scattering of polarized photons by molecules. Mol. Phys., 16:453-466, 1969. 
[27] L. D. Barron and A. D. Buckingham. Rayleigh and Raman scattering from optically active molecules. Mol. Phys., 20:1111-1119, 1971.

[28] L. D. Barron, M. P. Bogaard and A. D. Buckingham. Raman scattering of circularly polarized light by optically active molecules. J. Am. Chem. Soc., 95:603-605, 1973.

[29] W. Hug, S. Kint, G. F. Bailey and J. R. Scherer. Raman circular intensity differential spectroscopy. Spectra of (-)$\alpha$-pinene and (+)- $\alpha$-phenylethylamine. J. Am. Chem. Soc., 97:5589-5590, 1975.

[30] L. D. Barron and A. D. Buckingham. Vibrational optical activity. Chem. Phys. Lett., 492:199-213, 2010.

[31] R. A. Harris. Chiral fluctuations in achiral systems. J. Chem. Phys., 115:10577-10580, 2001.

[32] A. Einstein. Die Grundlage der allgemeinen Relativitätstheorie. Annalen der Physik, 354:769-822, 1916.

[33] N. B. Baranova and B. Ya. Zel'dovich. Two approaches to spatial dispersion in molecular scattering of light. Usp. Fiz. Nauk., 127:421-450, 1979.

[34] R. E. Raab. Magnetic multipole moments. Mol. Phys., 29:1323-1331, 1975.

[35] M. F. Maestre, C. Bustamante, T. L. Hayes, J. A. Subirana and I. Tinoco. Differential scattering of circularly polarized light by the helical sperm head from the octopus Eledone cirrhosa. Nature, 298:773-774, 1982.
[36] G. Züber, P. Wipf and D. N. Beratan. Exploring the optical activity tensor by anisotropic Rayleigh optical activity scattering. Chem. Phys. Chem., 9:265-271, 2008.

[37] R. P. Cameron and S. M. Barnett. Optical activity in the scattering of structured light. Phys. Chem. Chem. Phys., 16:25819-25829, 2014.

[38] R. P. Cameron, J. B. Götte, S. M. Barnett and A. M. Yao. Chirality and the angular momentum of light. Proc. Roy. Soc. A, 375:20150433, 2017.

[39] L. Hecht and L. D. Barron. Rayleigh and Raman optical activity from chiral surfaces. Chem. Phys. Lett., 225:525530, 1994.

[40] J. Applequist. On the polarizability theory of optical radiation J. Phys. Chem., 58:4251-4259, 1973.

[41] D. L. Andrews. Rayleigh and Raman optical activity: an analysis of the dependence on scattering angle. J. Chem. Phys., 72:4141-4144, 1980.

[42] R. P. Cameron and J. P. Cotter. Relativistic properties of a molecule: energy, linear momentum, angular momentum and boost momentum to order $1 / c^{2}$. J. Phys. B: At. Mol. Opt. Phys., 51:105101, 2018.

[43] O. L. de Lange and R. E. Raab. Multipole Theory in Electromagnetism: Classical, Quantum and Symmetry Aspects, with Applications. Oxford University Press, 2005. 